\title{
Biphasic enantioselective olefin epoxidation using Tropos Dibenzoazepinium catalysts
}

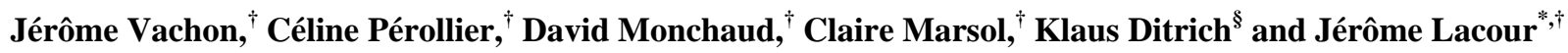

† Département de Chimie Organique, Université de Genève, quai Ernest-Ansermet 30, CH-1211 Genève 4, Switzerland $\S$ BASF Aktiengesellschaft, Forschung Feinchemikalien \& Biokatalyse, 67056 Ludwigshafen, Germany

Jerome.Lacour@chiorg.unige.ch

\section{Supporting Information}

1. General methods and Materials $\quad$ S2

2. NMR data of 11a S3

3. NMR and CD data of [7a][9] S6

4. NMR data of 11b $\quad$ S10

5. NMR and CD data of [7b][9 S12

6. NMR data of 11c $\quad$ S16

7. NMR and CD data of [7c][9] S18

8. NMR data of 11d $\quad$ S22

9. NMR and CD data of [7d][9] S24

$\begin{array}{ll}\text { 10. NMR data of 11e } & \text { S28 }\end{array}$

11. NMR and CD data of [7e][9] S31

12. NMR data of $\left[\mathrm{Me}_{2} \mathrm{NH}_{2}\right][9] \quad$ S35

13. Determination of the enantiomeric excess of the epoxide of olefin $12 \quad$ S38

14. Determination of the enantiomeric excess of the epoxide of olefin $13 \quad$ S39

15. Determination of the enantiomeric excess of the epoxide of olefin $14 \quad S 40$

16. Determination of the enantiomeric excess of the epoxide of olefin 15

17. Determination of the enantiomeric excess of the epoxide of olefin $16 \quad$ S42 
1. General Methods and Materials: Most of the reactions were carried out under dry $\mathrm{N}_{2}$ or Ar by means of an inert gas/vacuum double manifold line and standard Schlenk techniques with magnetic stirring, unless otherwise stated. Solvents were dried and distilled prior to use: toluene was freshly distilled from sodium, dichloromethane and hexane were freshly distilled from Calcium hydride, diethylether and tetrahydrofuran from sodium-benzophenone ketyl diethylether. $\mathrm{CHCl}_{3}$, $\mathrm{CH}_{2} \mathrm{Cl}_{2}, \mathrm{CDCl}_{3}$ and $\mathrm{CD}_{2} \mathrm{Cl}_{2}$ were filtered on basic alumina. All chemicals were purchased an used as received or purified according to standard literature procedures. Analytical thin-layer chromatography (TLC) was performed with TLC plates pre-coated with silica gel or $0.25 \mathrm{~mm}$ basic alumina ( $\mathrm{pH}=9.9$ ) plates. Visualization of the developed chromatogram was performed by UV/VIS detection. Unless otherwise specified, column chromatography (silica gel 60, $40 \mathrm{~mm}$ or basic alumina type 5016A) was performed in air and under pressure (0.1-0.3 bar). NMR spectra were recorded on a 300, 400 or $500 \mathrm{MHz}$ apparatus at room temperature. ${ }^{1} \mathrm{H}-\mathrm{NMR}$ : chemical shifts are given in ppm relative to $\mathrm{Me}_{4} \mathrm{Si}$ with the solvent resonance used as the internal standard. ${ }^{31} \mathrm{P}-\mathrm{NMR}$ : chemical shifts were reported in ppm relative to $\mathrm{H}_{3} \mathrm{PO}_{4} \cdot{ }^{13} \mathrm{C}-\mathrm{NMR}$ : chemical shifts were given in ppm relative to $\mathrm{Me}_{4} \mathrm{Si}$, with the solvent resonance used as the internal standard $\left(\mathrm{CDCl}_{3} \delta 77.0 \mathrm{ppm}\right.$, $[\mathrm{D}]_{6} \mathrm{DMSO} \delta 39.5 \mathrm{ppm}, \mathrm{CD}_{2} \mathrm{Cl}_{2} \delta$ 53.8). IR spectra were recorded with a FT-IR spectrometer using a diamond head sampling and are reported in wavenumbers $\left(\mathrm{cm}^{-1}\right)$. Melting points (mp) were measured in open capillary tubes and were uncorrected. UV spectra were measured with a $1.0 \mathrm{~cm}$ quartz cell; $\lambda$ max are given in nm and molar adsorption coefficient $\varepsilon$ in $\mathrm{cm}^{-1} \cdot \mathrm{dm}^{3} \cdot \mathrm{mol}^{-1}$. Circular dichroism spectra were measured with a $1.0 \mathrm{~cm}$ quartz cell; 1 are given in nm and molar circular dichroic absorptions $(\Delta \varepsilon)$ in $\mathrm{cm}^{2} \cdot \mathrm{mmol}^{-1}$. Optical rotations were measured on a polarimeter in a thermostated $\left(20^{\circ} \mathrm{C}\right) 10.0 \mathrm{~cm}$ long microcell with high pressure lamps of sodium or mercury and are reported as follows: ( $c(\mathrm{~g} / 100 \mathrm{ml})$, solvent). Chiral stationary phase (CSP) chromatography was performed with a GC chromatograph using a Hydrodex $\beta$-3P column (25m x 0.25 mm). Retention times $\left(\mathrm{t}_{\mathrm{R}}\right)$ are given in minutes (min). CSP-HPLC analyses were performed using a binary pump, an autosampler, a column thermostat, a diode array detector and an OD-H column (250 x $4.6 \mathrm{~mm})$. 
${ }^{1} \mathrm{H}$ NMR $\left(400 \mathrm{MHz}, \mathrm{CDCl}_{3}\right)$

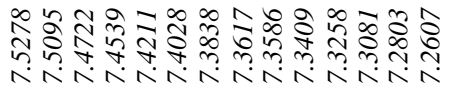

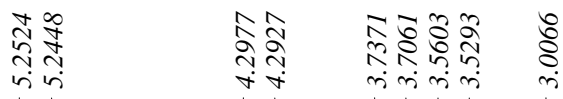

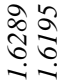
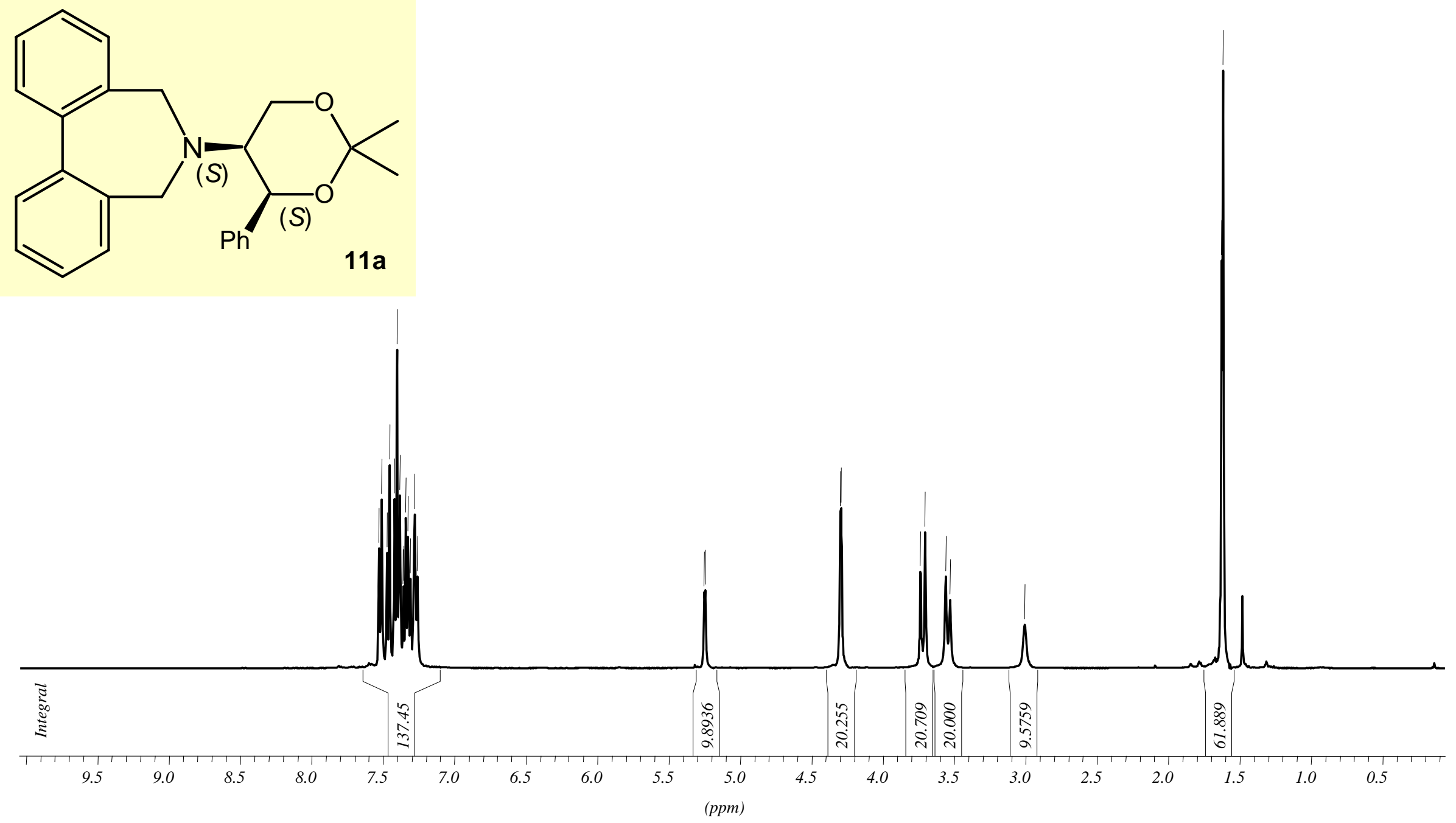


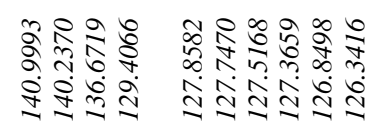

$\frac{1}{2}$

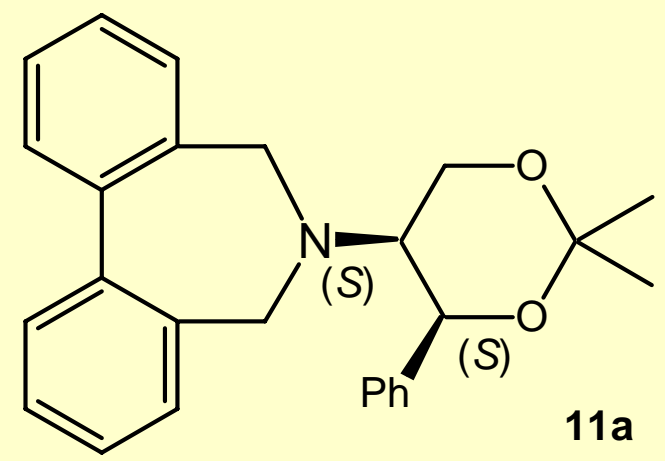

${ }^{13} \mathrm{C}$ NMR $\left(100 \mathrm{MHz}, \mathrm{CDCl}_{3}\right)$

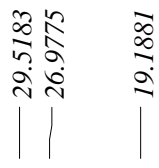

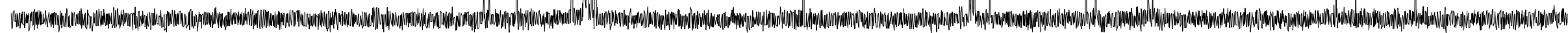

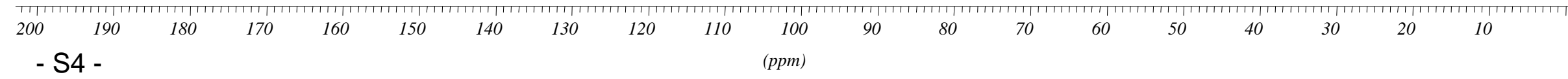


${ }^{13} \mathrm{C}$ NMR $\left(100 \mathrm{MHz}, \mathrm{CDCl}_{3}\right)$, DEPT135

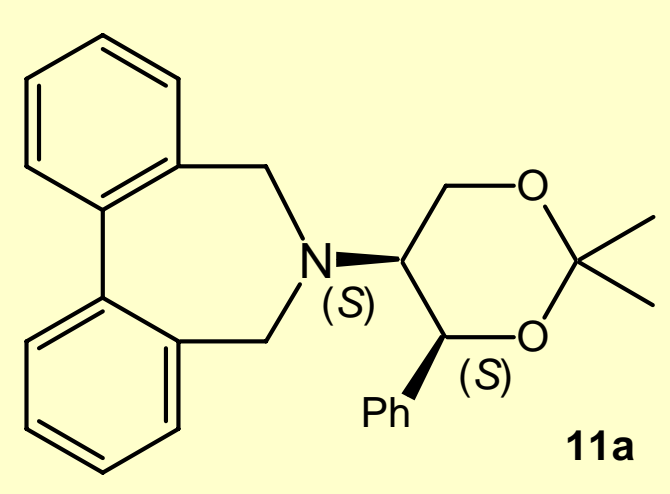

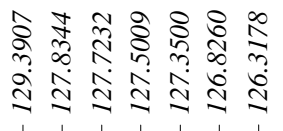

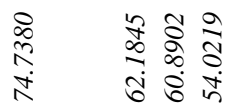

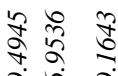

จे ขึ 


\section{6-N-(S)-(2,2-Dimethyl-4-(S)-phenyl-[1,3]dioxan-5-yl)-5H-dibenzo[c,e]azepinium.rac TT}

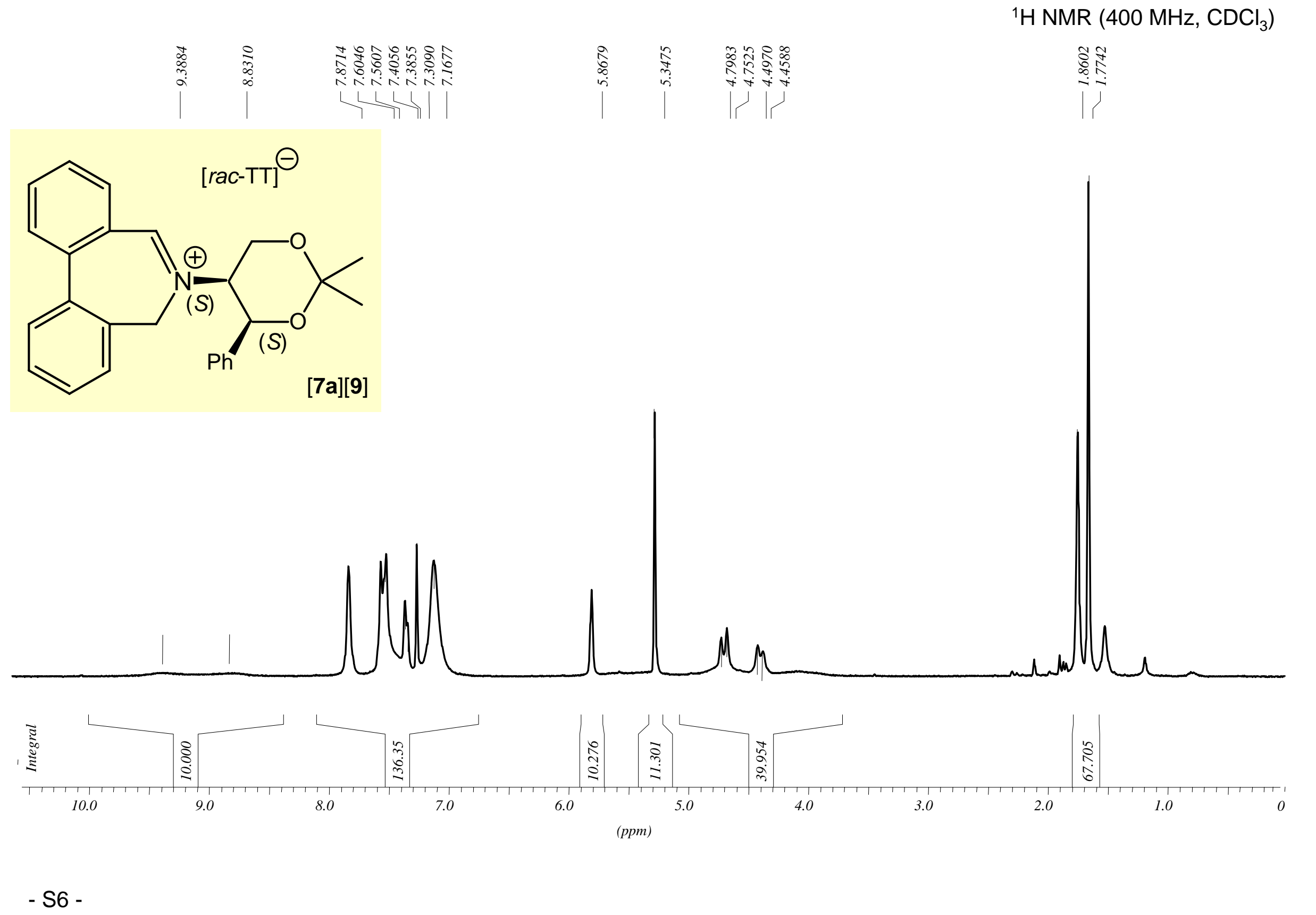


署

${ }^{31} \mathrm{P}$ NMR (162 MHz, CDCl ${ }_{3}$ )

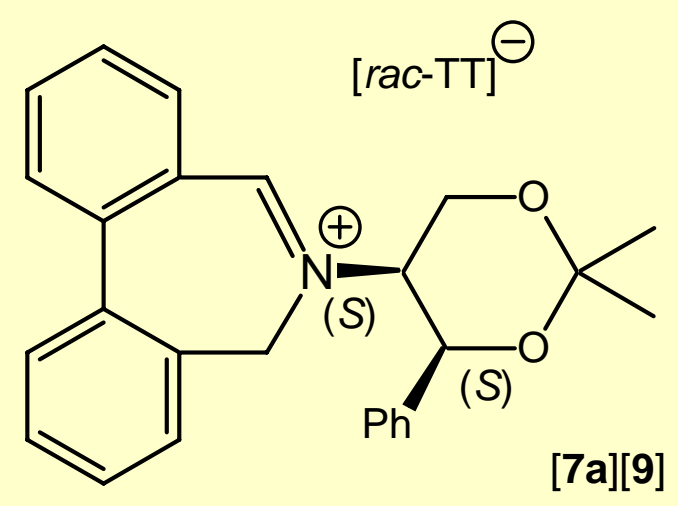




\section{6-N-(S)-(2,2-Dimethyl-4-(S)-phenyl-[1,3]dioxan-5-yl)-5H-dibenzo[c,e]azepinium.rac TT}

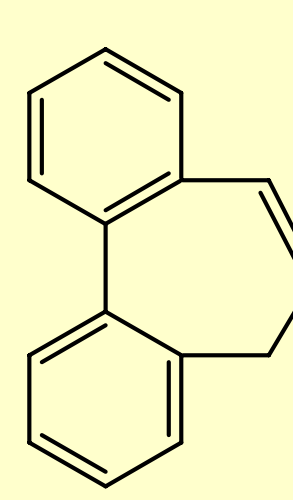

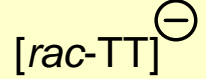

${ }^{1} \mathrm{H}$ NMR $\left(500 \mathrm{MHz}, \mathrm{CD}_{2} \mathrm{Cl}_{2}\right)$

Region
$\delta 10.0-0.0$ ppm

273

263

253

243

233

9.0

8.0

7.0

6.0

5.0

4.0

3.0

2.0

1.0

- S8 -

(рpm) 


\section{6-N-(S)-(2,2-Dimethyl-4-(S)-phenyl-[1,3]dioxan-5-yl)-5H-dibenzo[c,e]azepinium.rac TT}
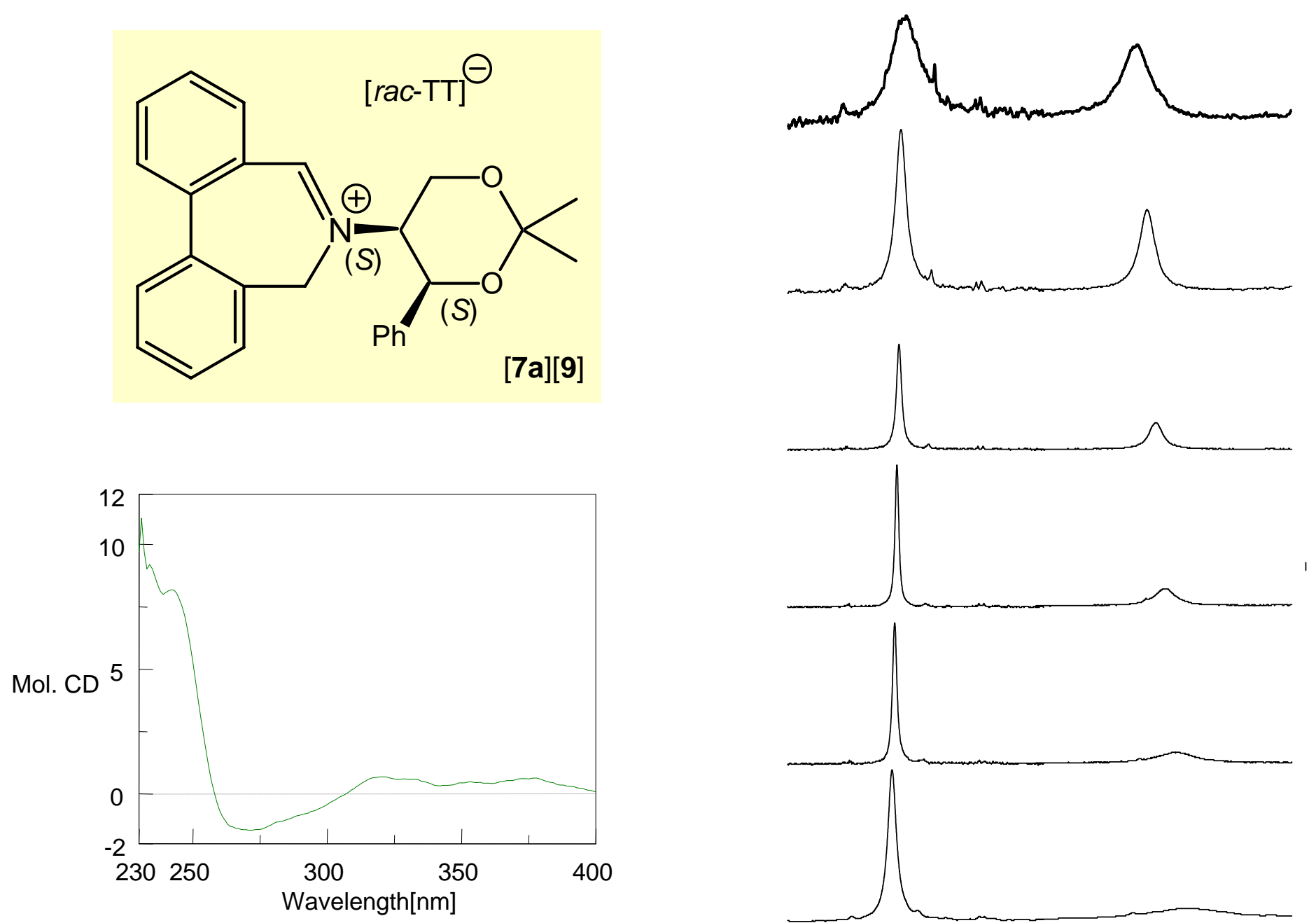

${ }^{1} \mathrm{H}$ NMR (500 MHz, $\mathrm{CD}_{2} \mathrm{Cl}_{2}$ )

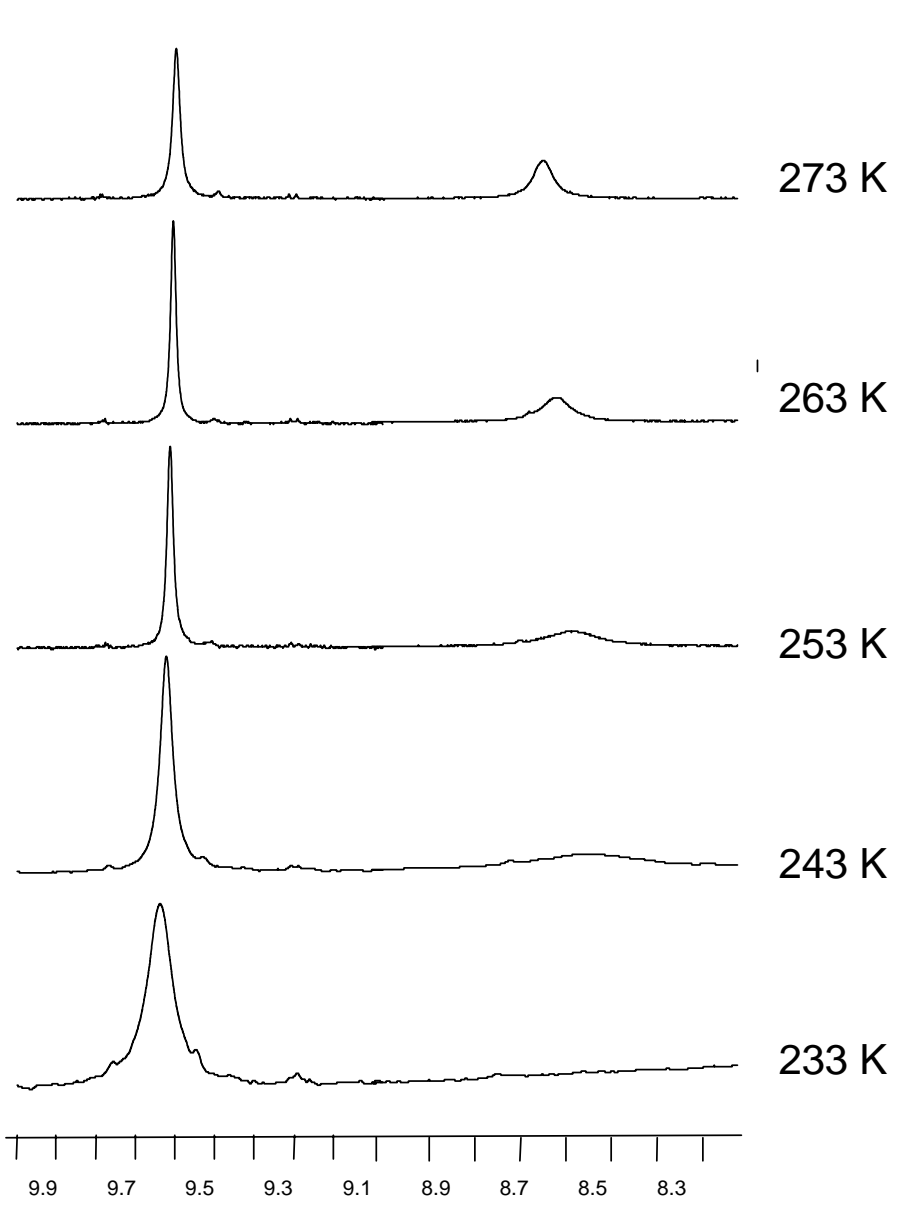


${ }^{1} \mathrm{H}$ NMR $\left(400 \mathrm{MHz}, \mathrm{CDCl}_{3}\right)$

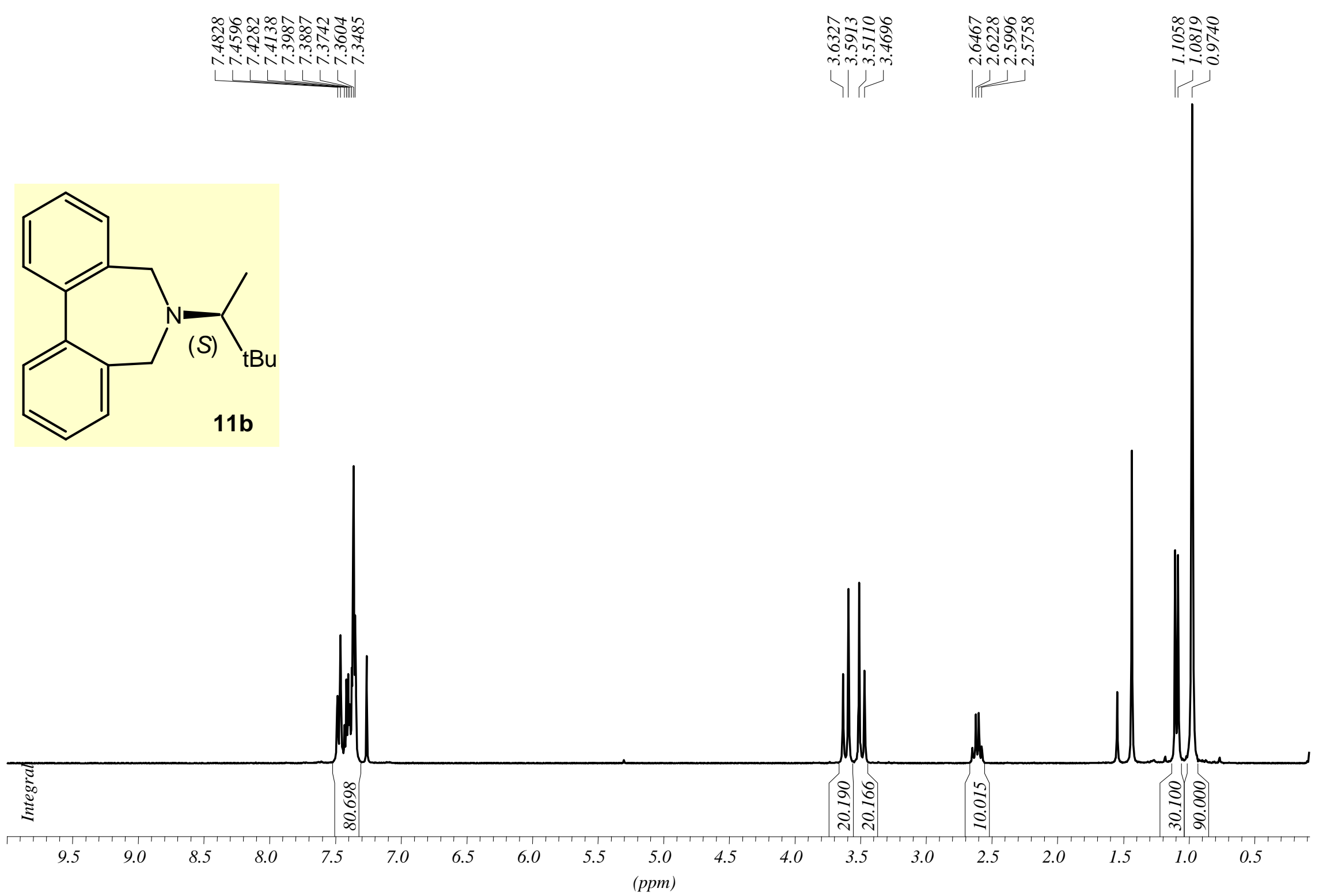


${ }^{13} \mathrm{C}$ NMR $\left(100 \mathrm{MHz}, \mathrm{CDCl}_{3}\right)$
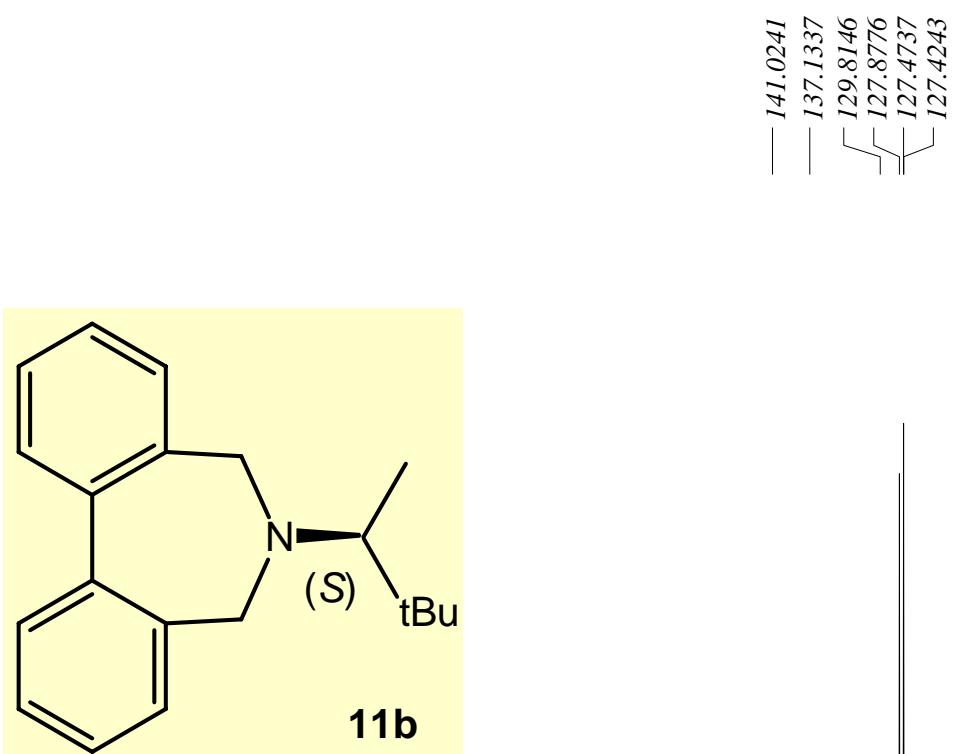

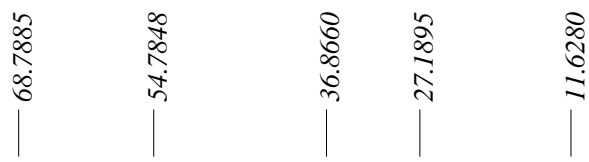

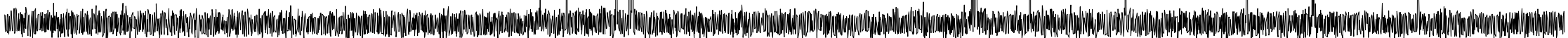

160

140

120 (ppm)

80

60

40

20

- S11 - 
${ }^{1} \mathrm{H}$ NMR (400 MHz, CDCl ${ }_{3}$ )
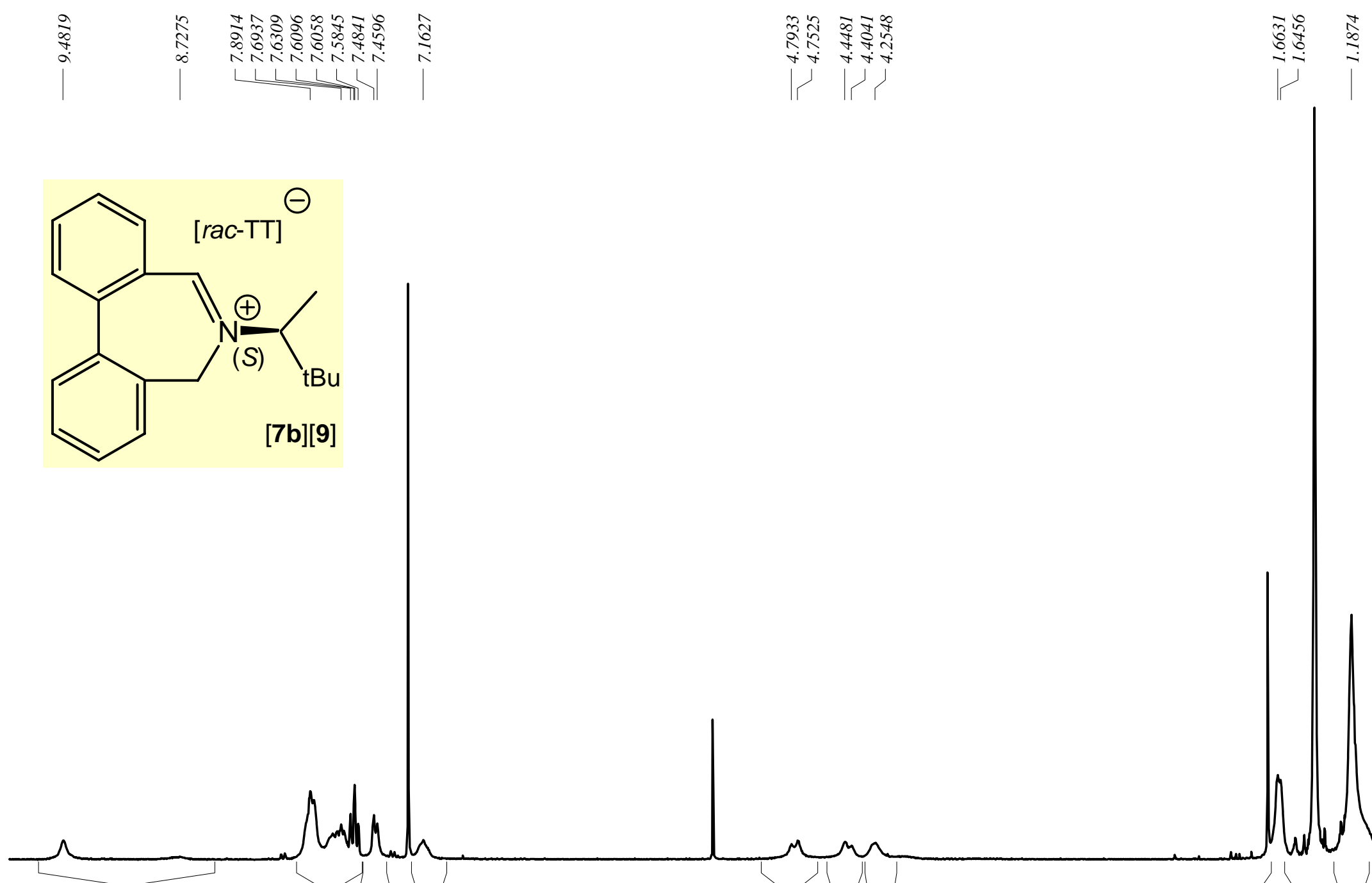

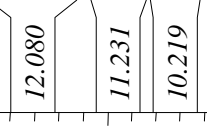

4.0

3.5
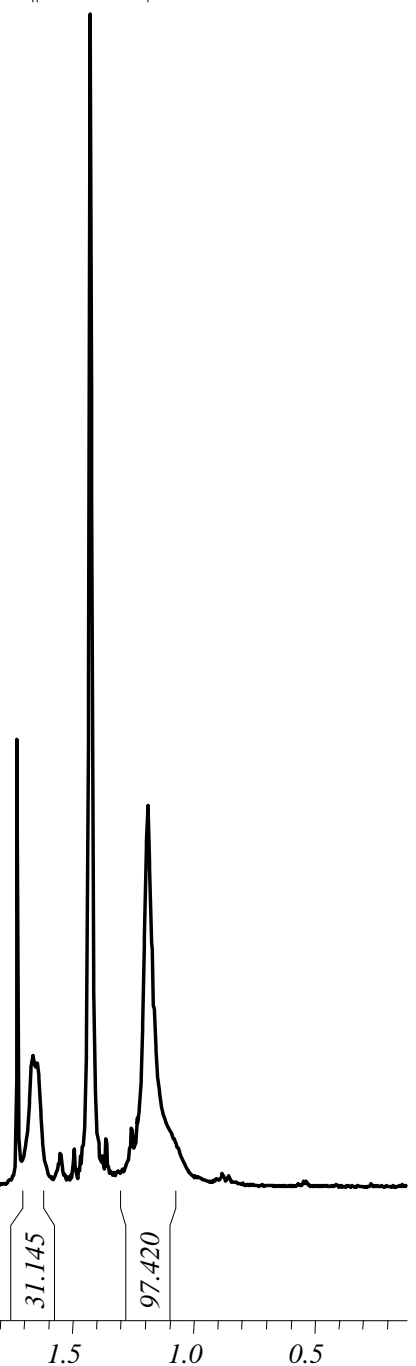
${ }^{31} \mathrm{P}$ NMR $\left(162 \mathrm{MHz}, \mathrm{CDCl}_{3}\right)$

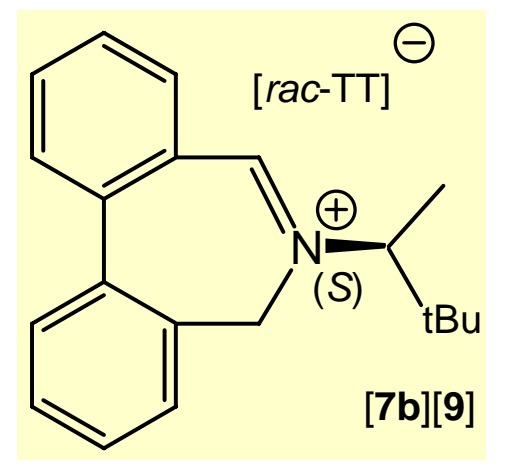

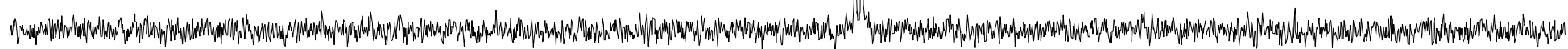

\begin{tabular}{|c|c|c|c|c|c|c|c|c|c|}
\hline-72 & -74 & -76 & -78 & $\begin{array}{c}-80 \\
(p p m)\end{array}$ & -82 & -84 & -86 & -88 & -90 \\
\hline
\end{tabular}




\section{6-N-((S)-1,2,2-trimethypropyl)-5H-dibenz[c,e]azepinium.rac TT}

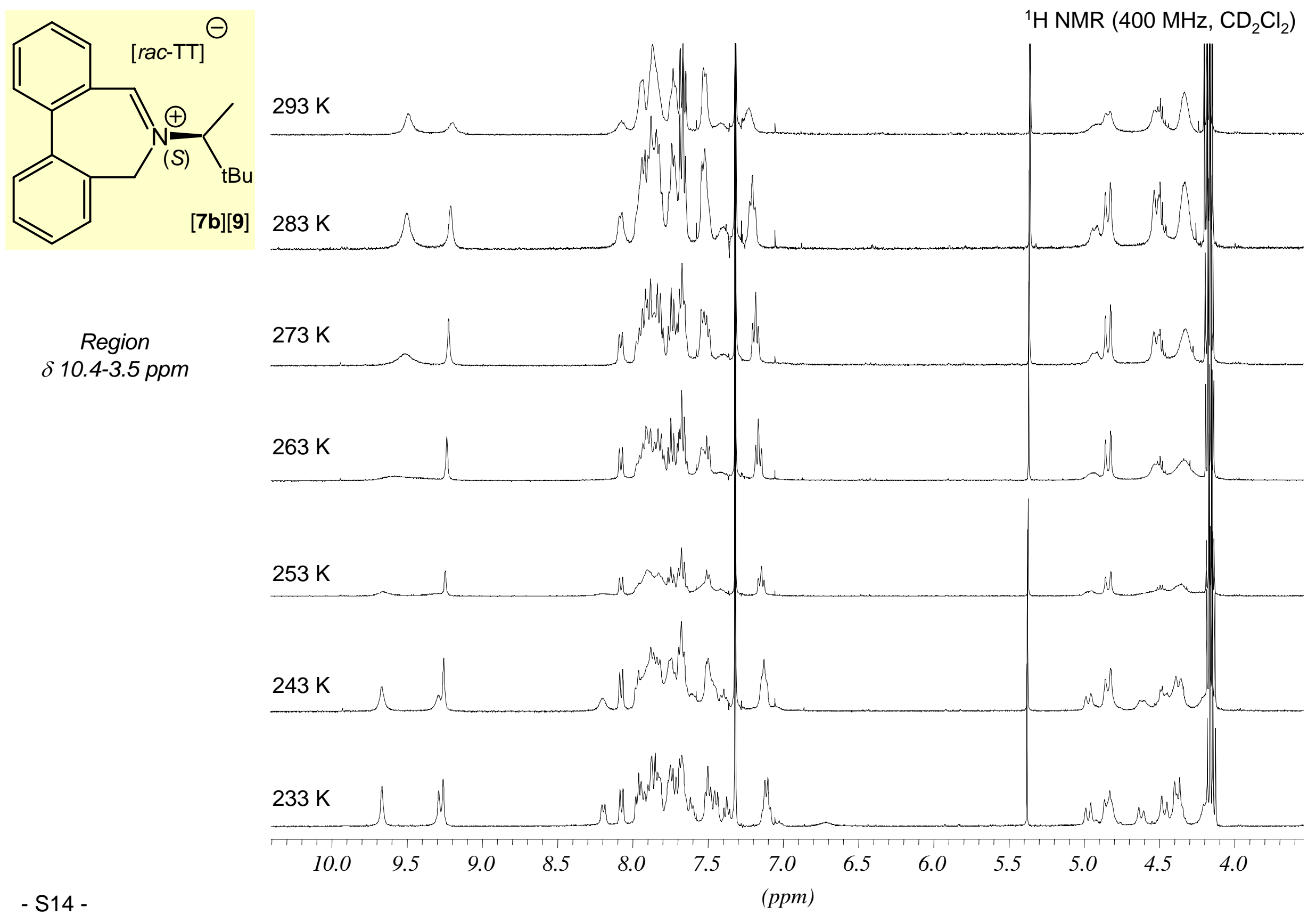


${ }^{1} \mathrm{H}$ NMR (400 MHz, $\mathrm{CD}_{2} \mathrm{Cl}_{2}$ )

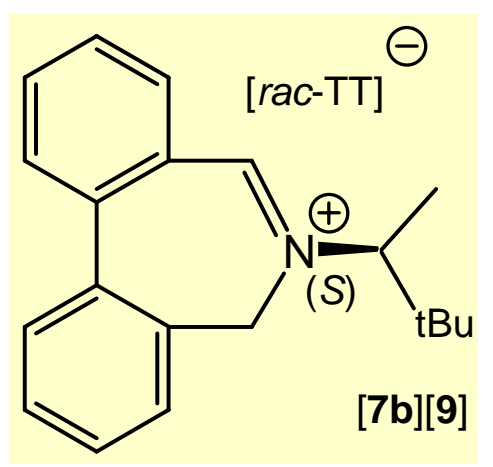

$298 \mathrm{~K}$

Region

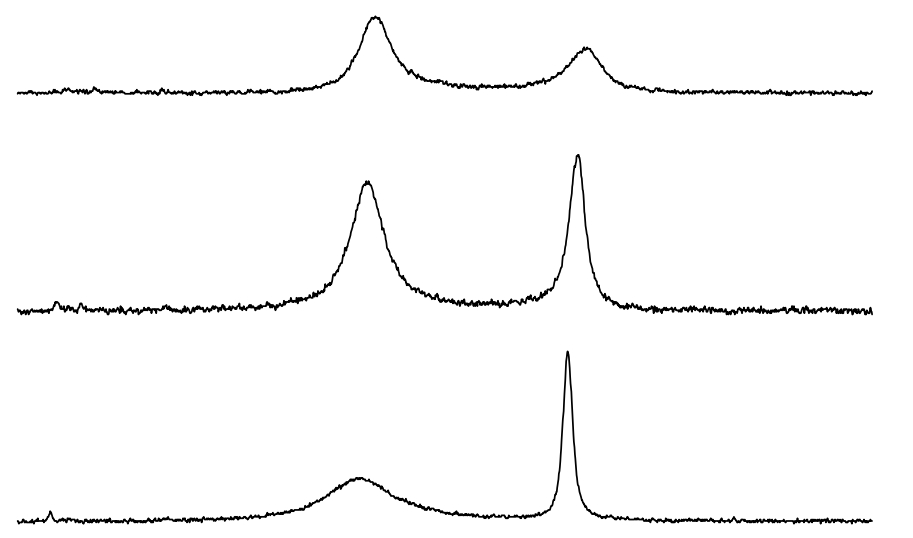

$\delta$ 9.9-8.9 ppm
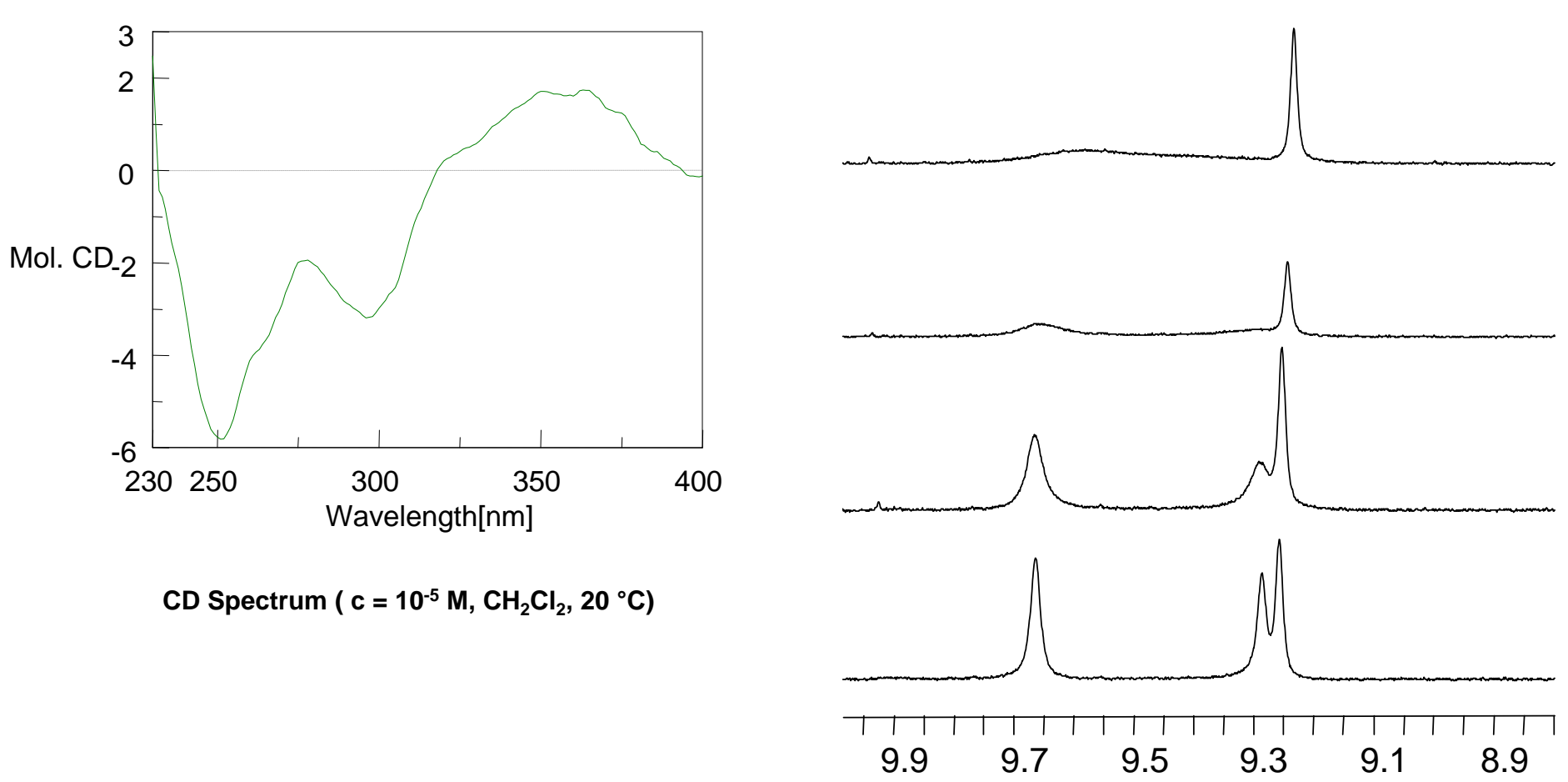

$273 \mathrm{~K}$

$263 \mathrm{~K}$

$253 \mathrm{~K}$

$243 \mathrm{~K}$

CD Spectrum ( $\mathrm{c}=10^{-5} \mathrm{M}, \mathrm{CH}_{2} \mathrm{Cl}_{2}, 20^{\circ} \mathrm{C}$ )

$233 \mathrm{~K}$

- S15 - 
${ }^{1} \mathrm{H}$ NMR (400 MHz, $\left.\mathrm{CDCl}_{3}\right)$

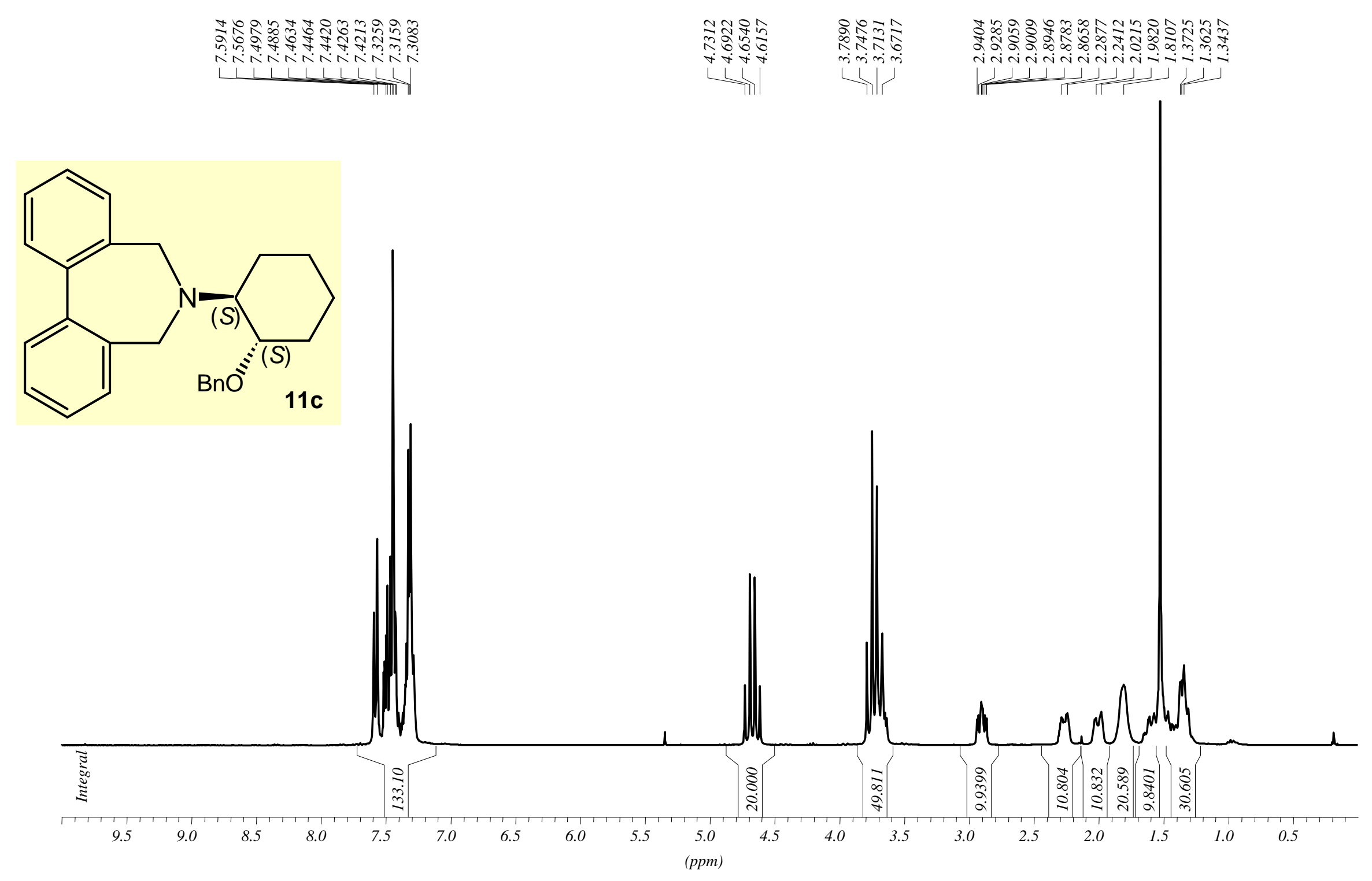


${ }^{13} \mathrm{C}$ NMR $\left(100 \mathrm{MHz}, \mathrm{CDCl}_{3}\right)$
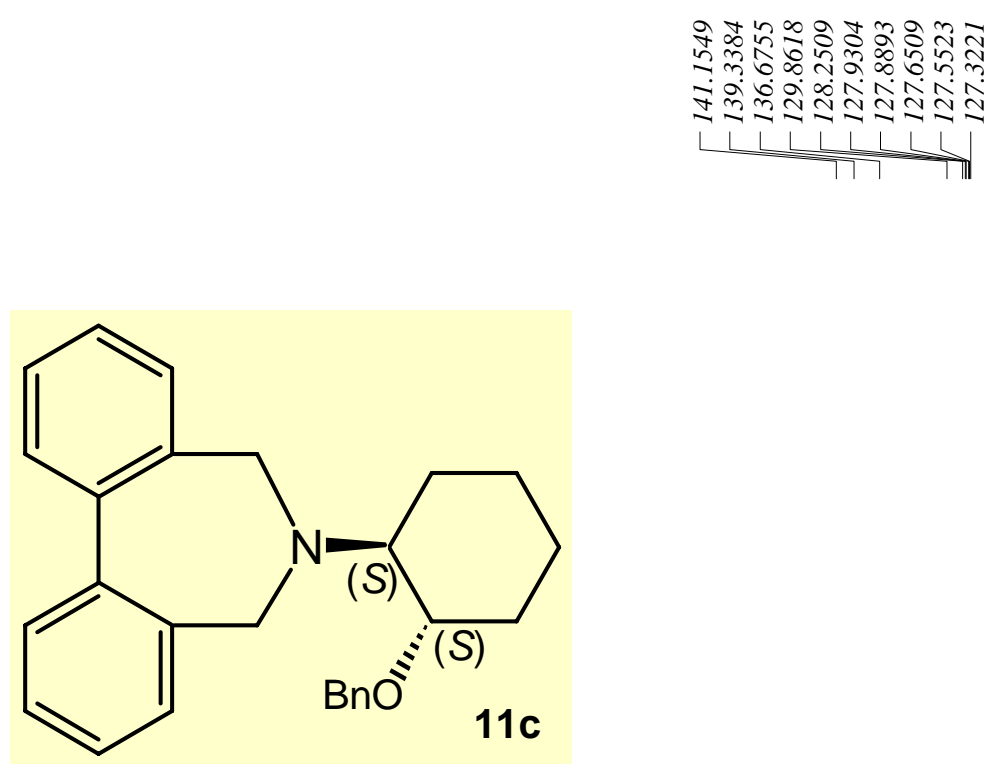

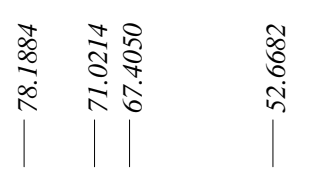

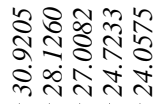

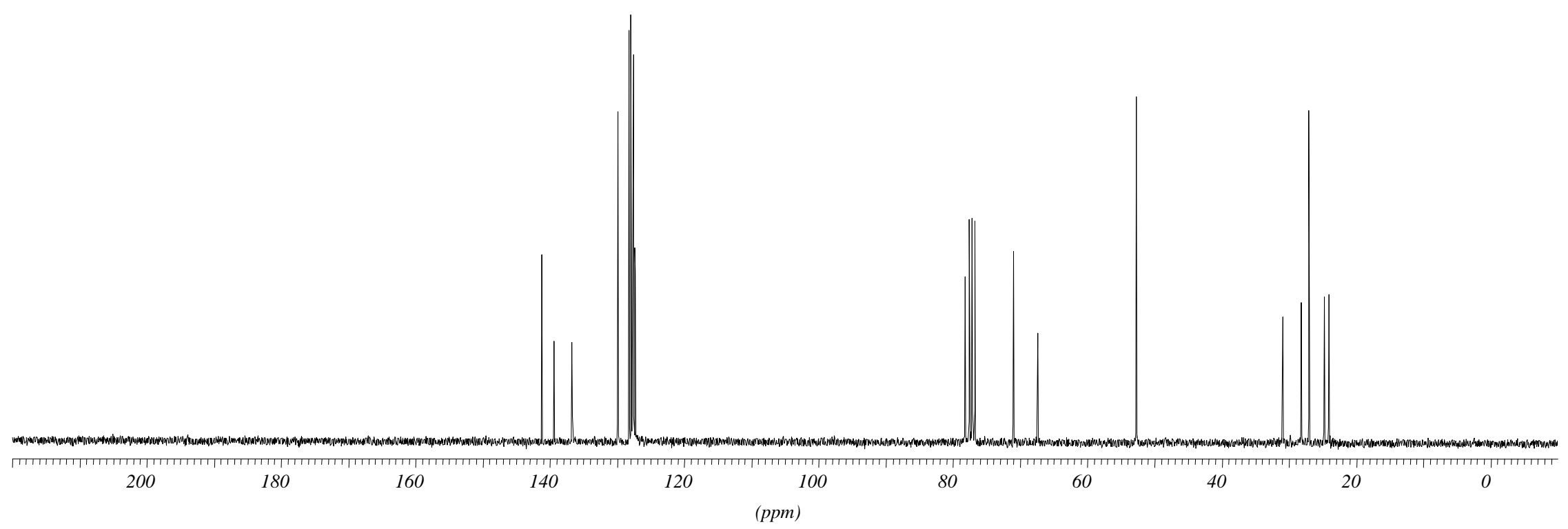


${ }^{1} \mathrm{H}$ NMR $\left(400 \mathrm{MHz}, \mathrm{CDCl}_{3}\right)$

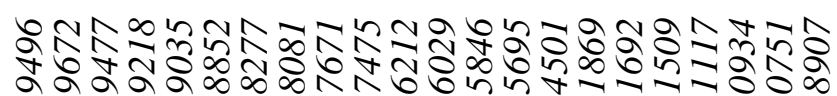

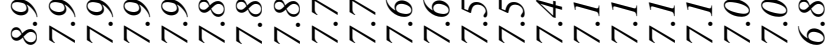
IIIIII
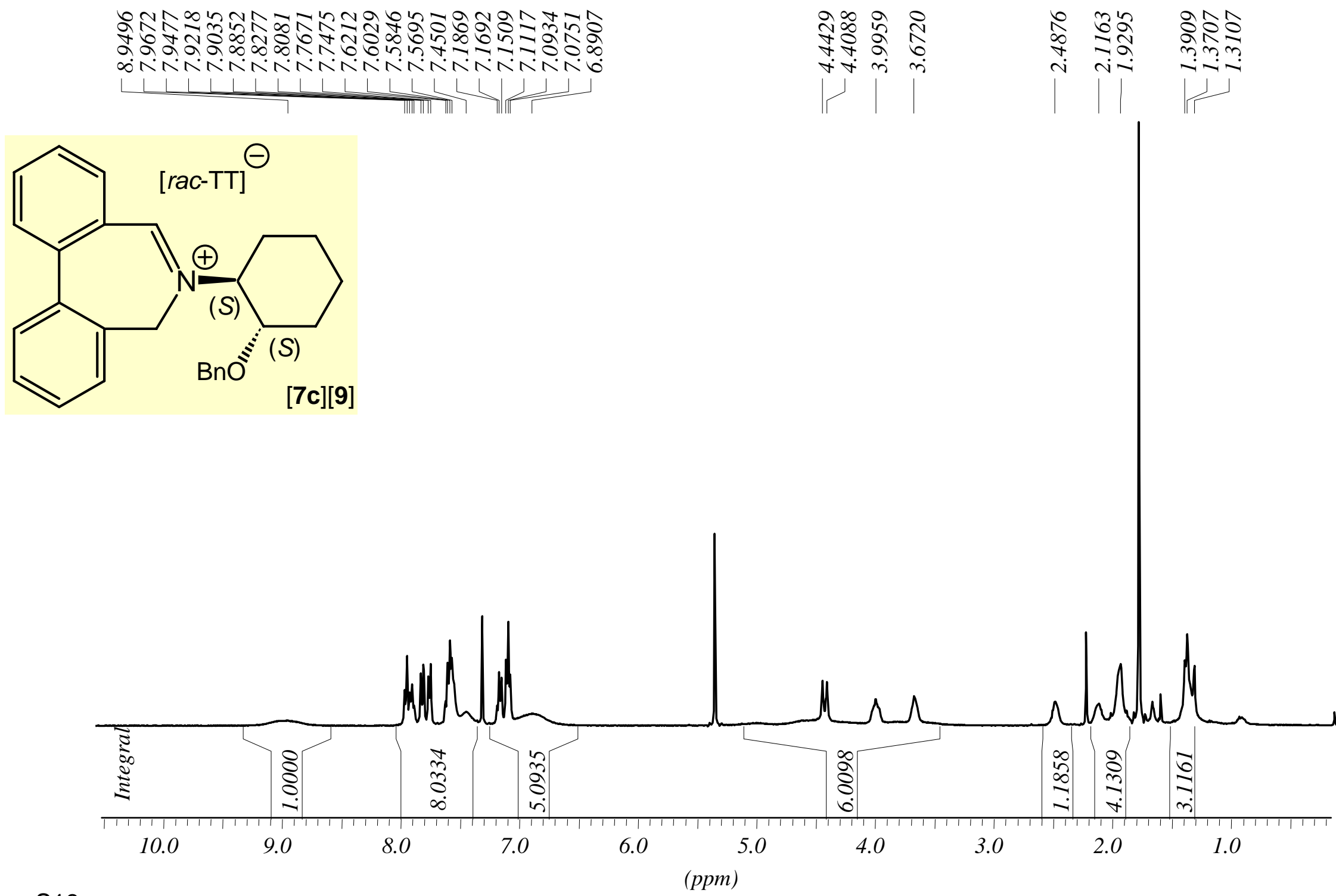

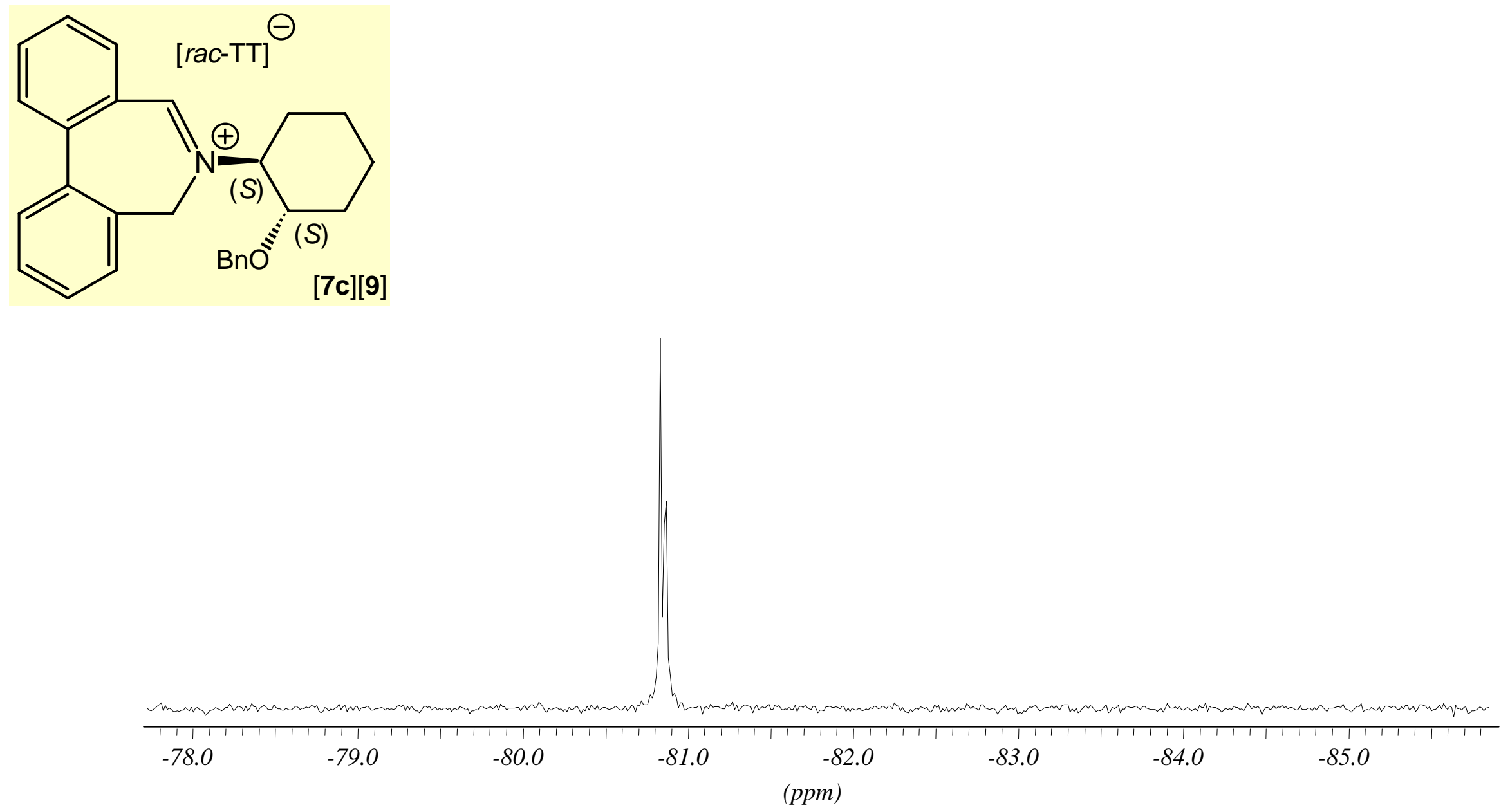


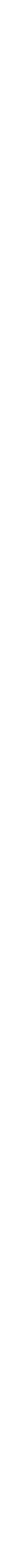




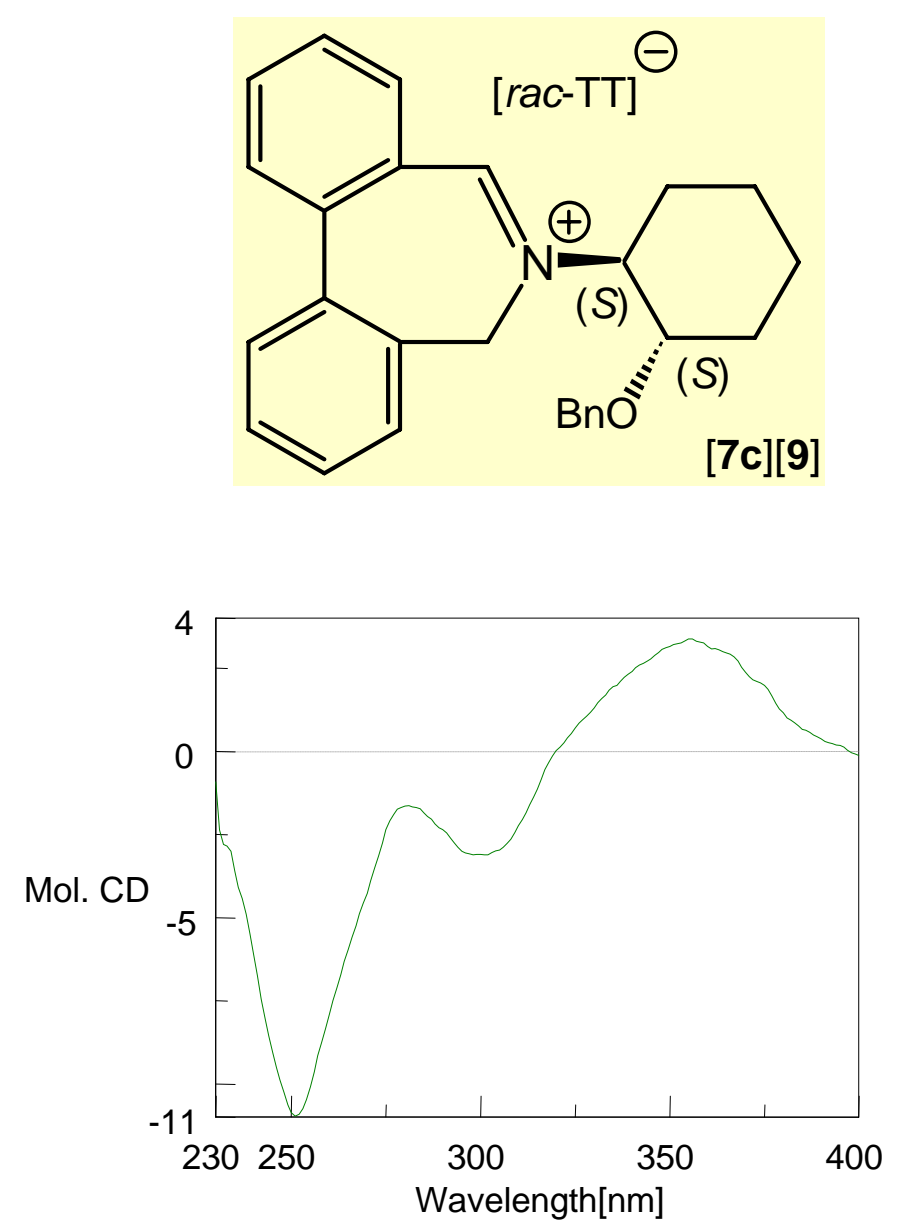

${ }^{1} \mathrm{H}$ NMR $\left(500 \mathrm{MHz}, \mathrm{CD}_{2} \mathrm{Cl}_{2}\right)$

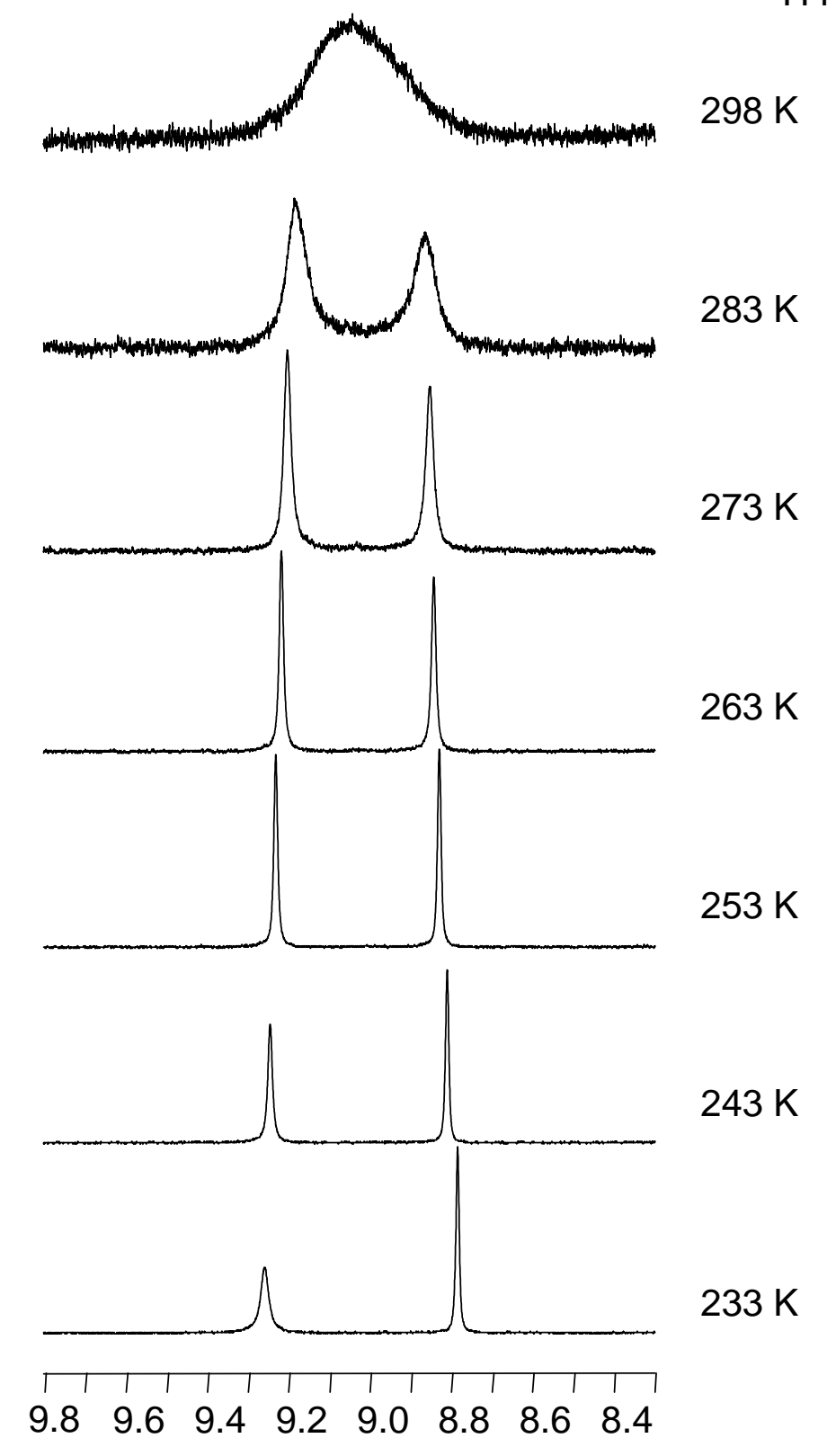


${ }^{1} \mathrm{H}$ NMR $\left(400 \mathrm{MHz}, \mathrm{CDCl}_{3}\right)$

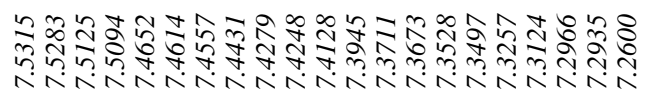

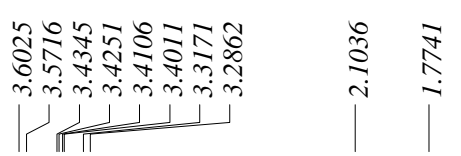

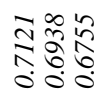
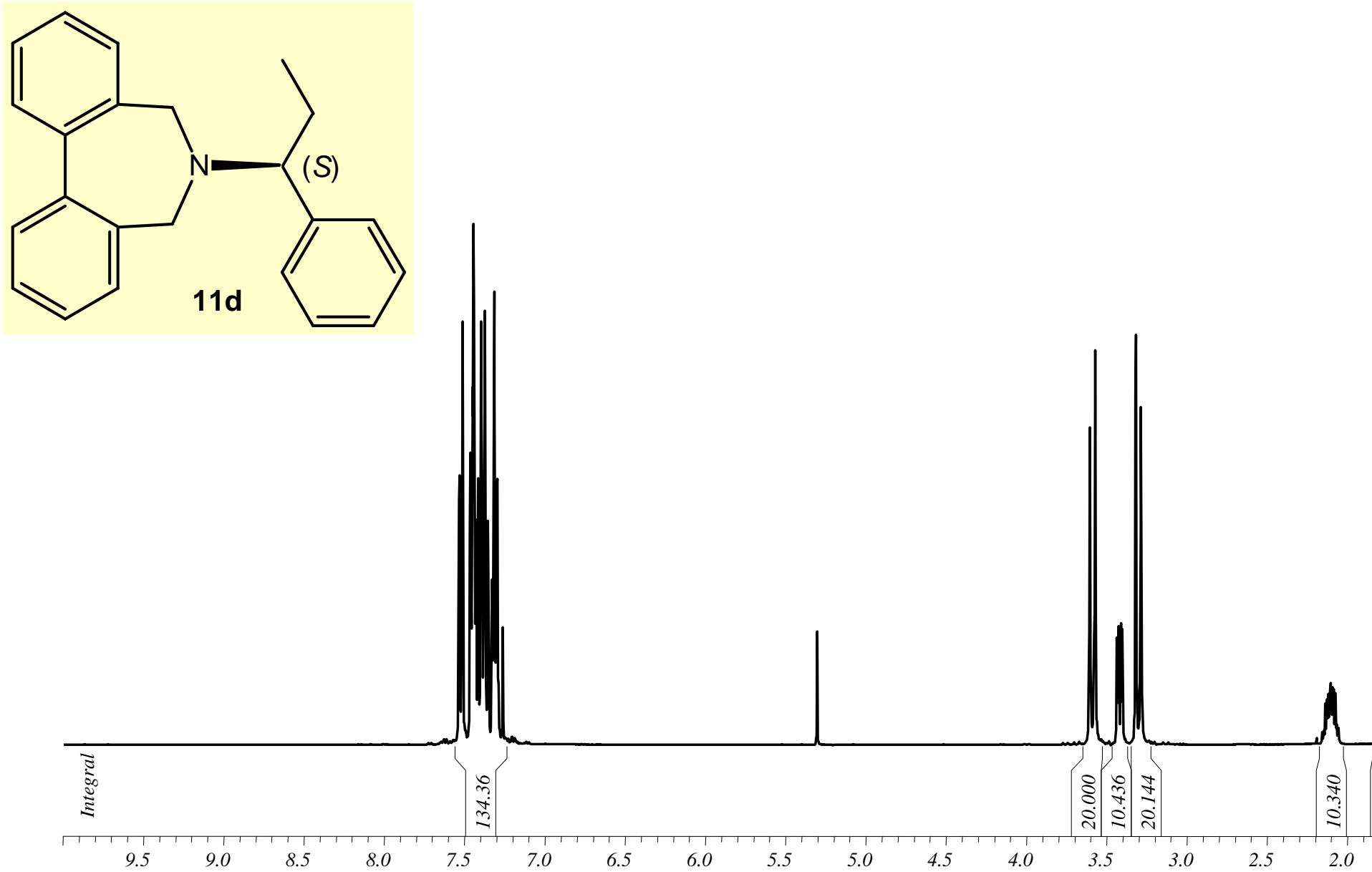
${ }^{13} \mathrm{C}$ NMR $\left(100 \mathrm{MHz}, \mathrm{CDCl}_{3}\right)$

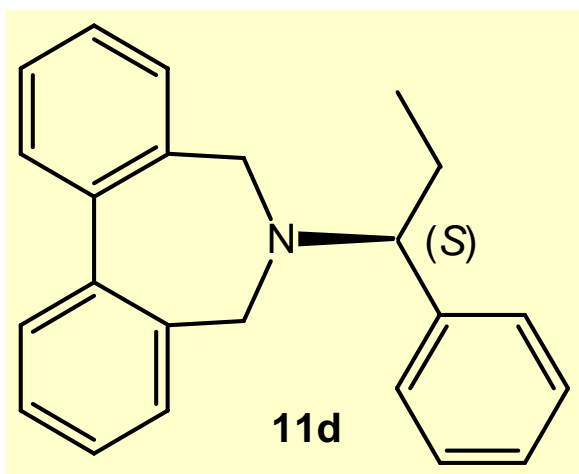

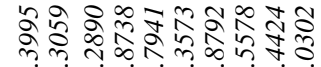

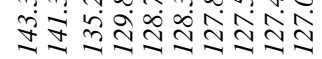

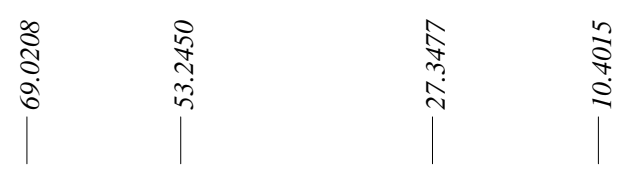


${ }^{1} \mathrm{H}$ NMR (400 MHz, $\mathrm{CDCl}_{3}$ )
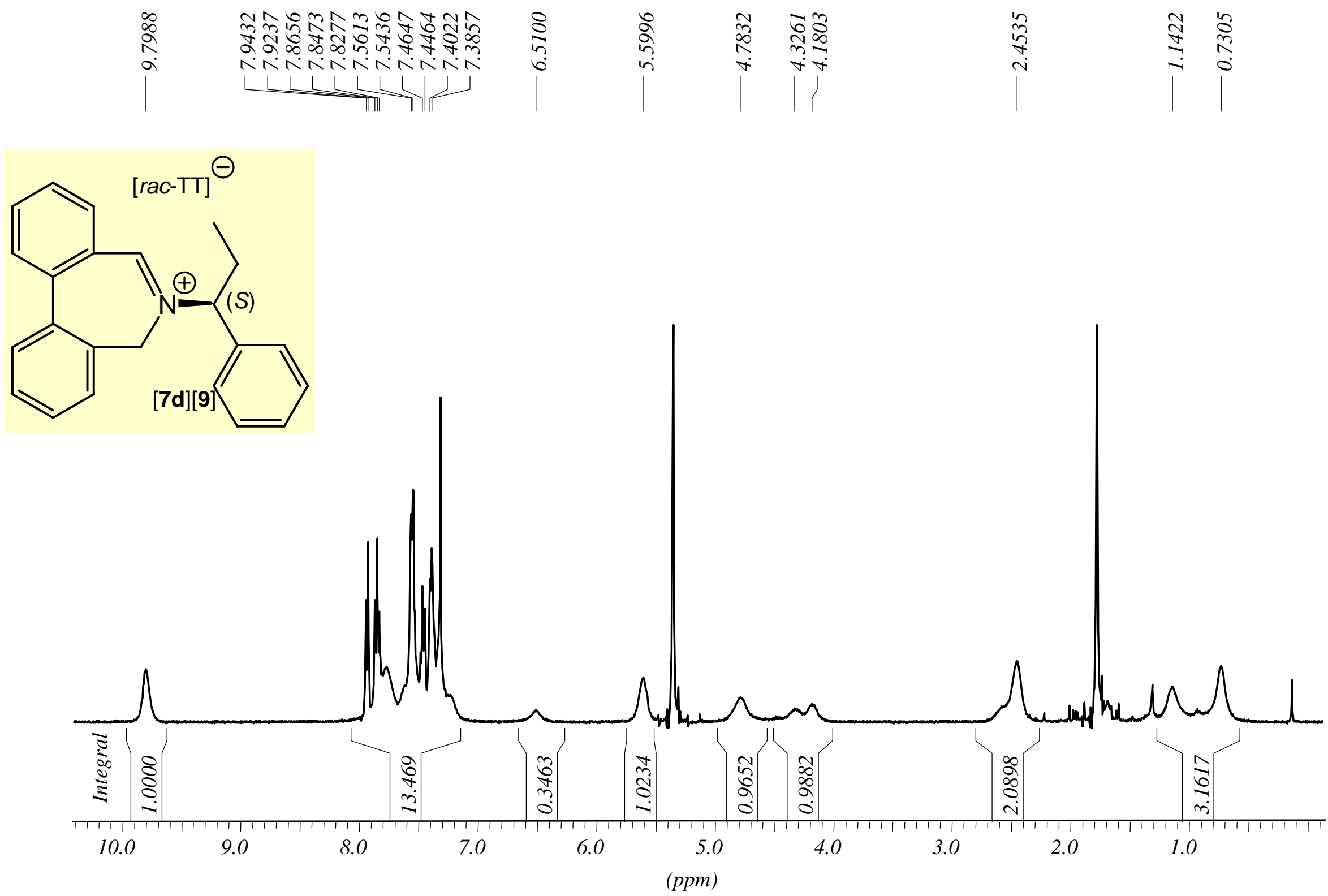

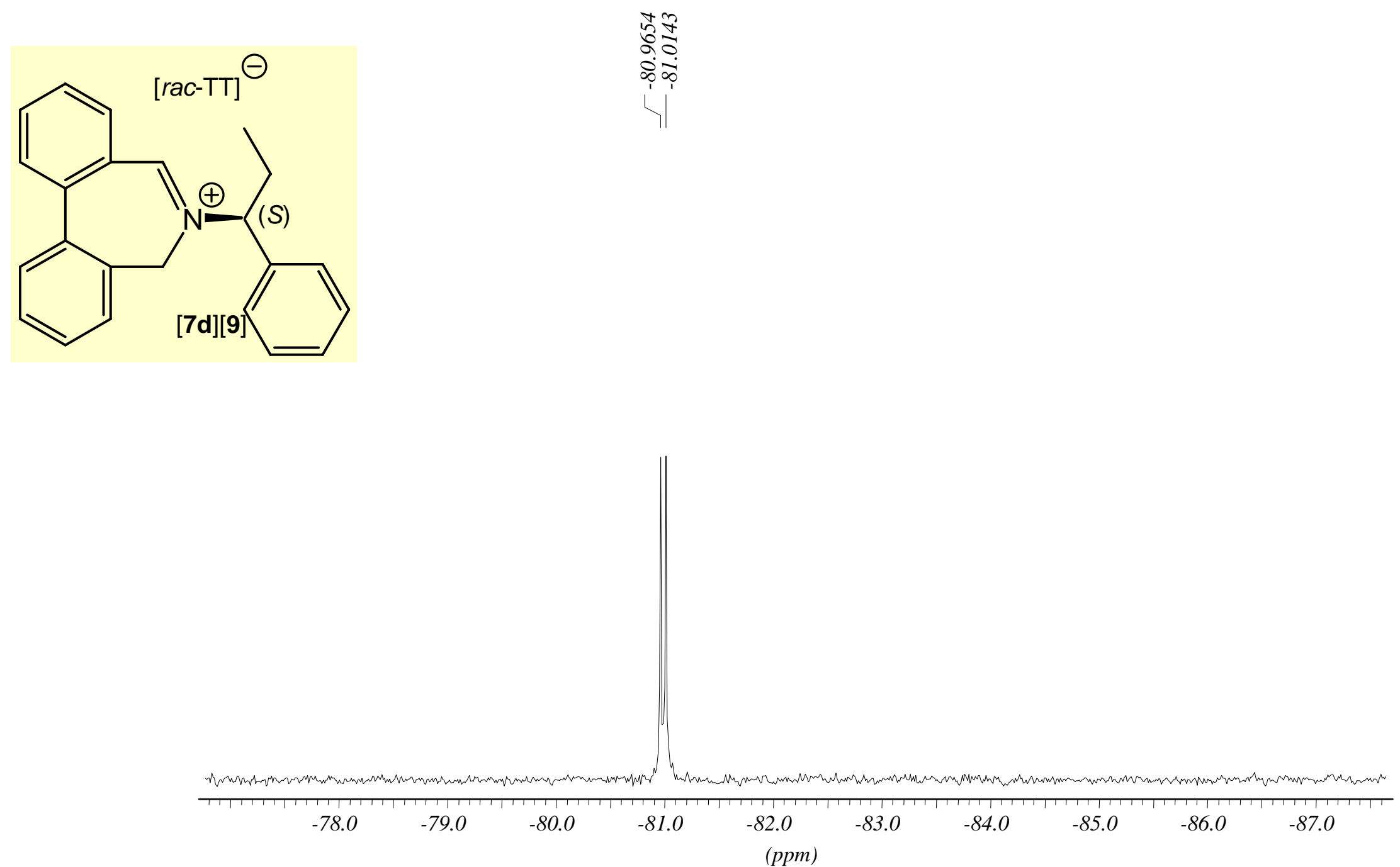


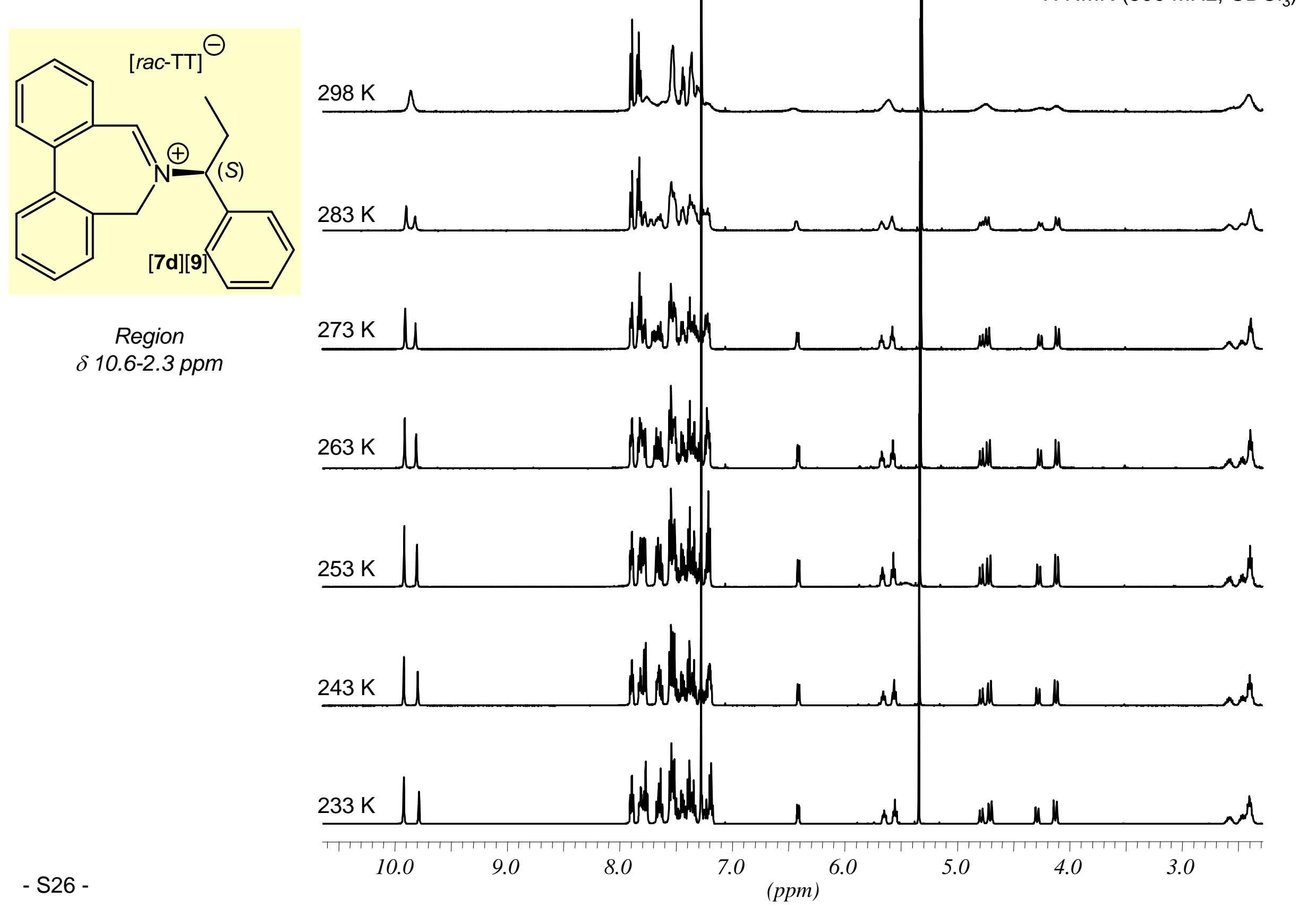



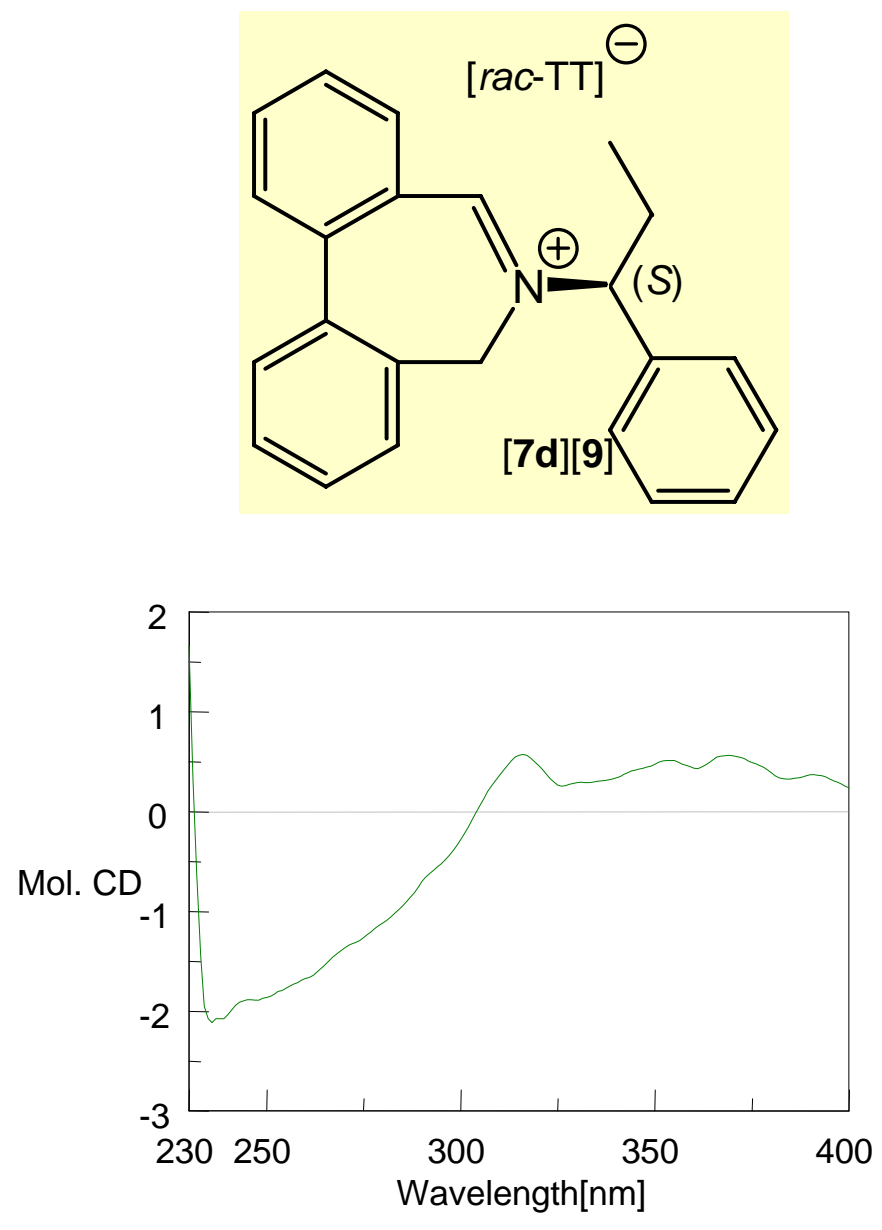

CD Spectrum ( $\mathrm{c}=10^{-5} \mathrm{M}, \mathrm{CH}_{2} \mathrm{Cl}_{2}, 20^{\circ} \mathrm{C}$ )
${ }^{1} \mathrm{H} \mathrm{NMR}\left(500 \mathrm{MHz}, \mathrm{CDCl}_{3}\right)$

Region

298 K $\delta$ 10.3-9.5 ppm

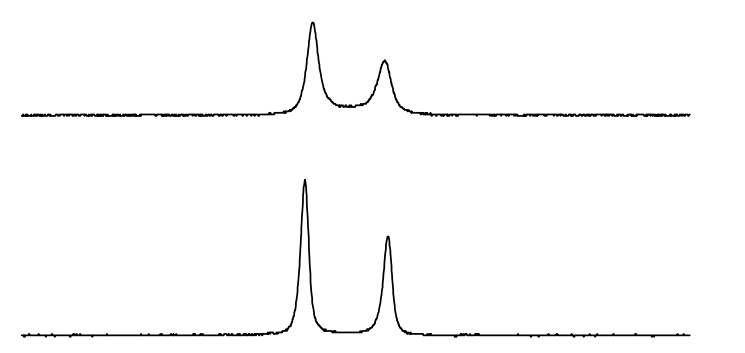

$283 \mathrm{~K}$

$273 \mathrm{~K}$

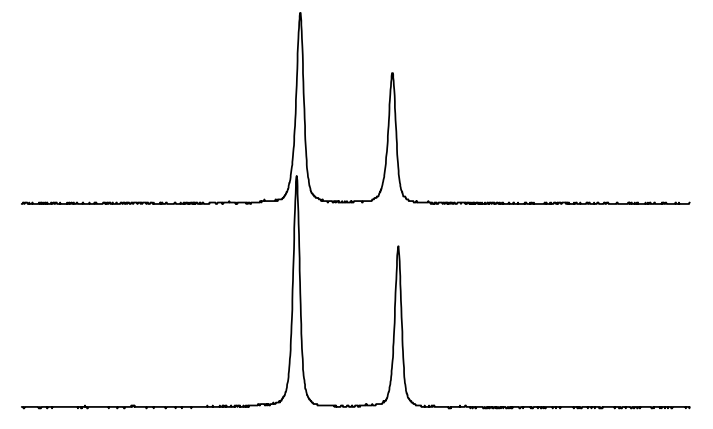

$263 \mathrm{~K}$

$253 \mathrm{~K}$
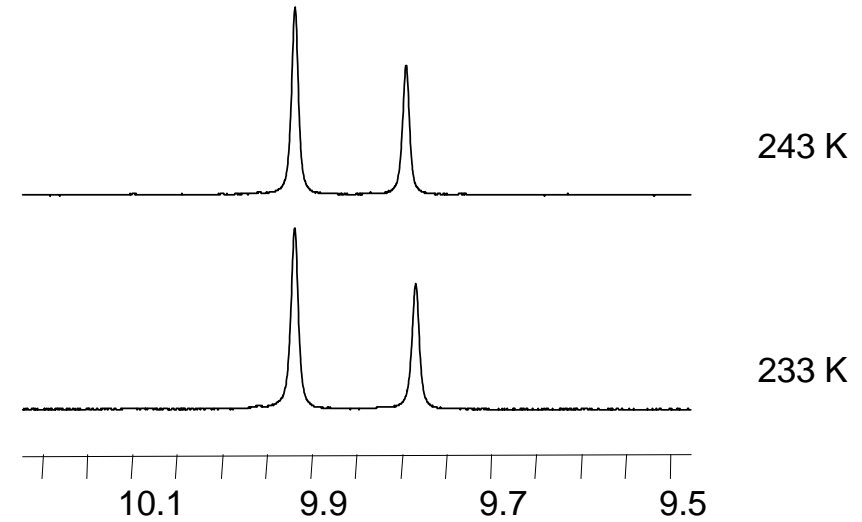

$233 \mathrm{~K}$ 
(20)<smiles>C[C@H](c1cccc2ccccc12)N1Cc2ccccc2-c2ccccc2C1</smiles>

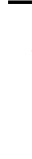

ธิ

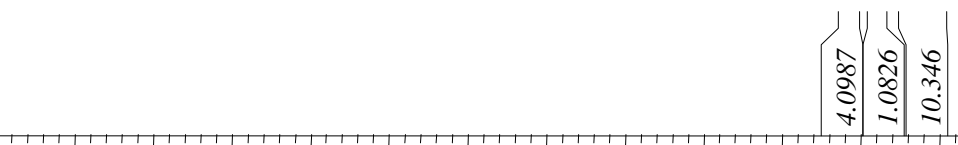

13.0

12.0

11.0

10.0

9.0

8.0

7.0
ppm)

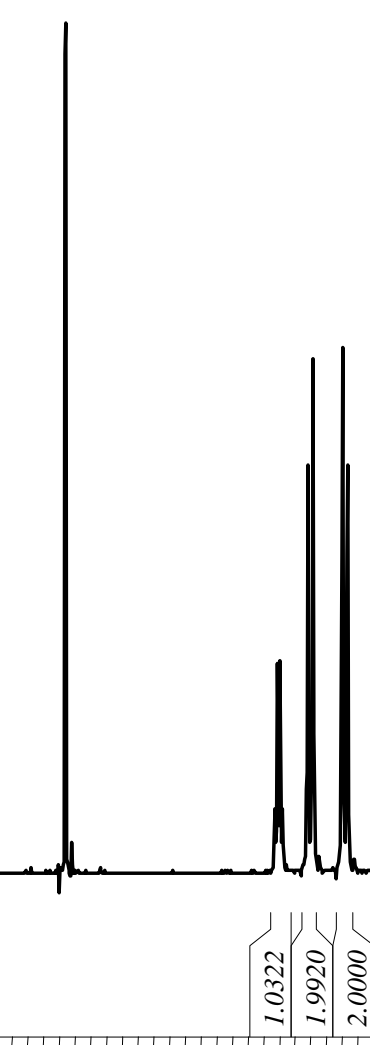

5.0

4.0

3.0

1.0 
${ }^{13} \mathrm{C}$ NMR $\left(100 \mathrm{MHz}, \mathrm{CDCl}_{3}\right)$, DEPT 135

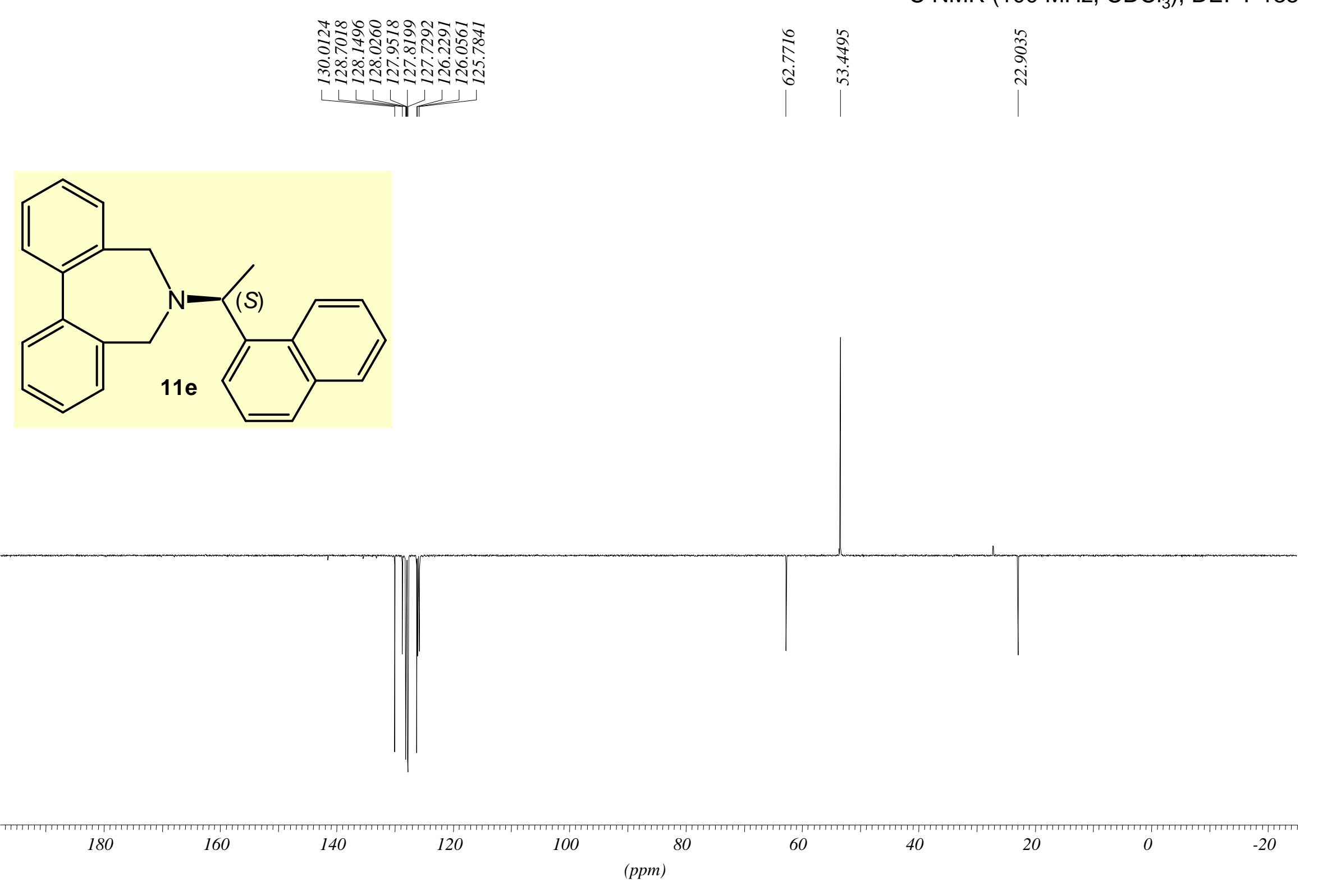

- S29 - 


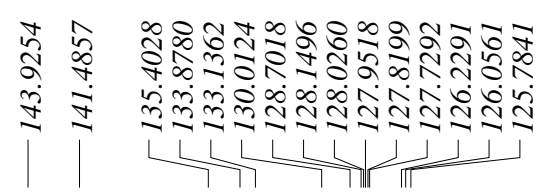

${ }^{13} \mathrm{C}$ NMR $\left(100 \mathrm{MHz}, \mathrm{CDCl}_{3}\right)$
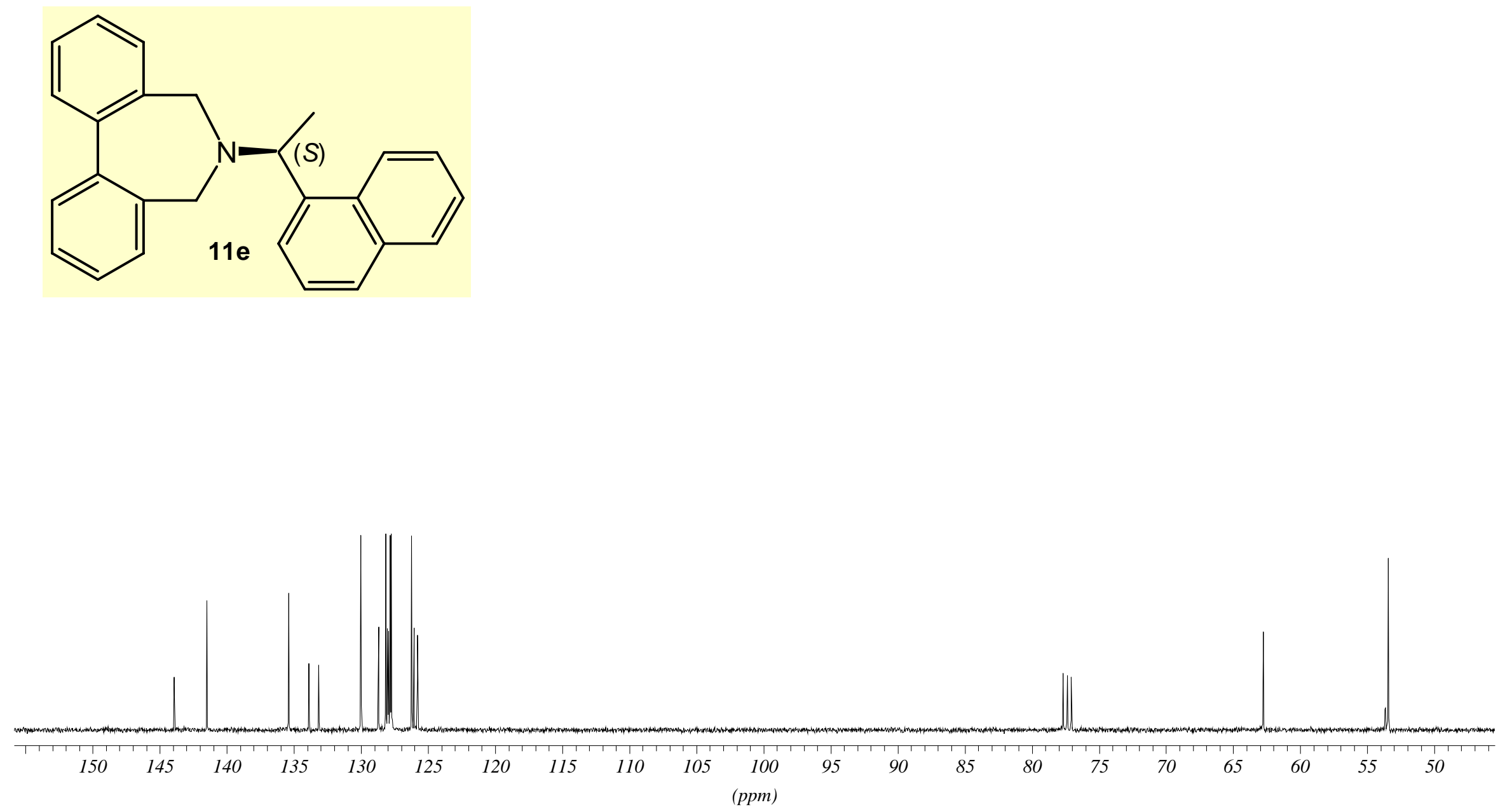


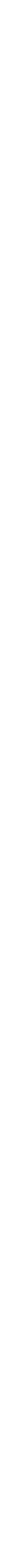


${ }^{31} \mathrm{P}$ NMR $\left(162 \mathrm{MHz}, \mathrm{CDCl}_{3}\right)$
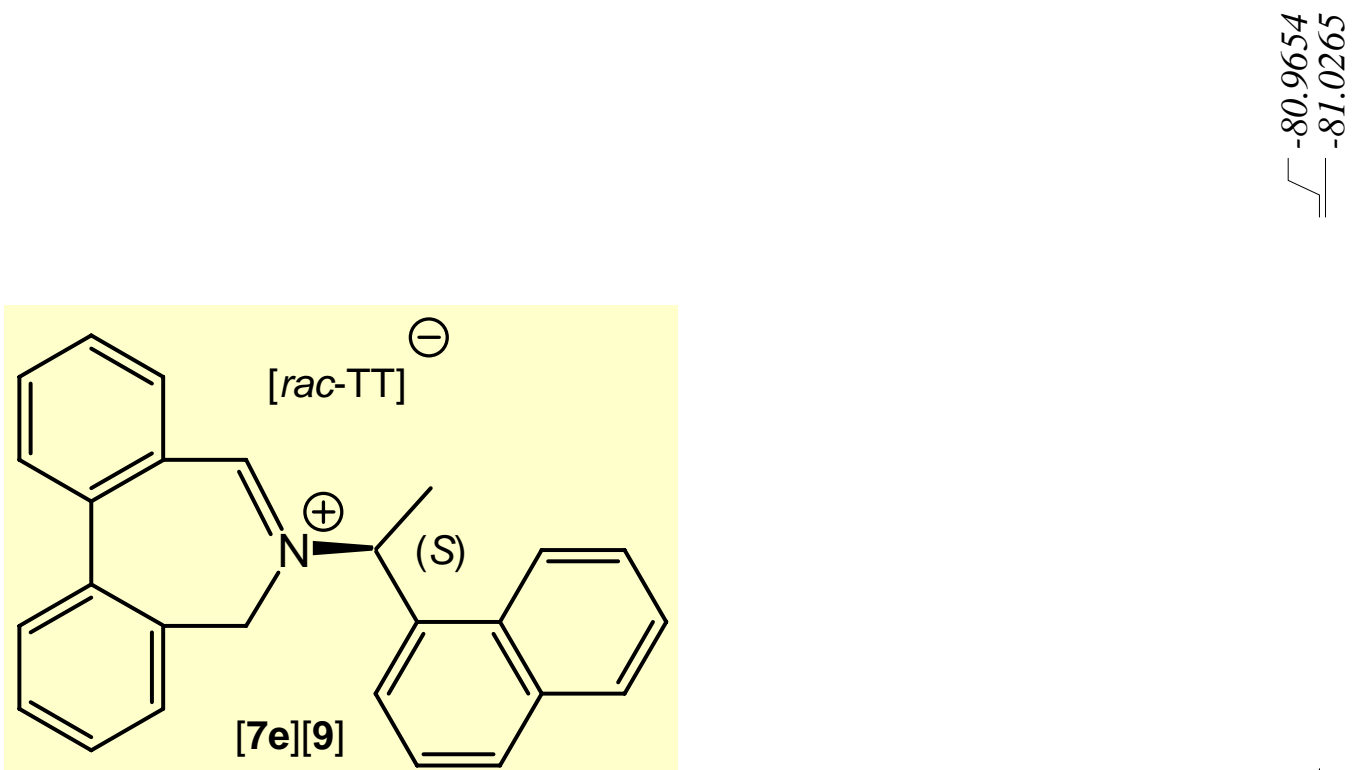

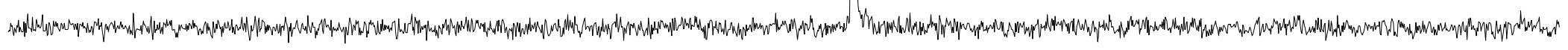

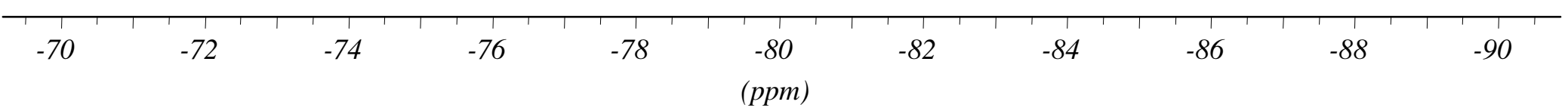




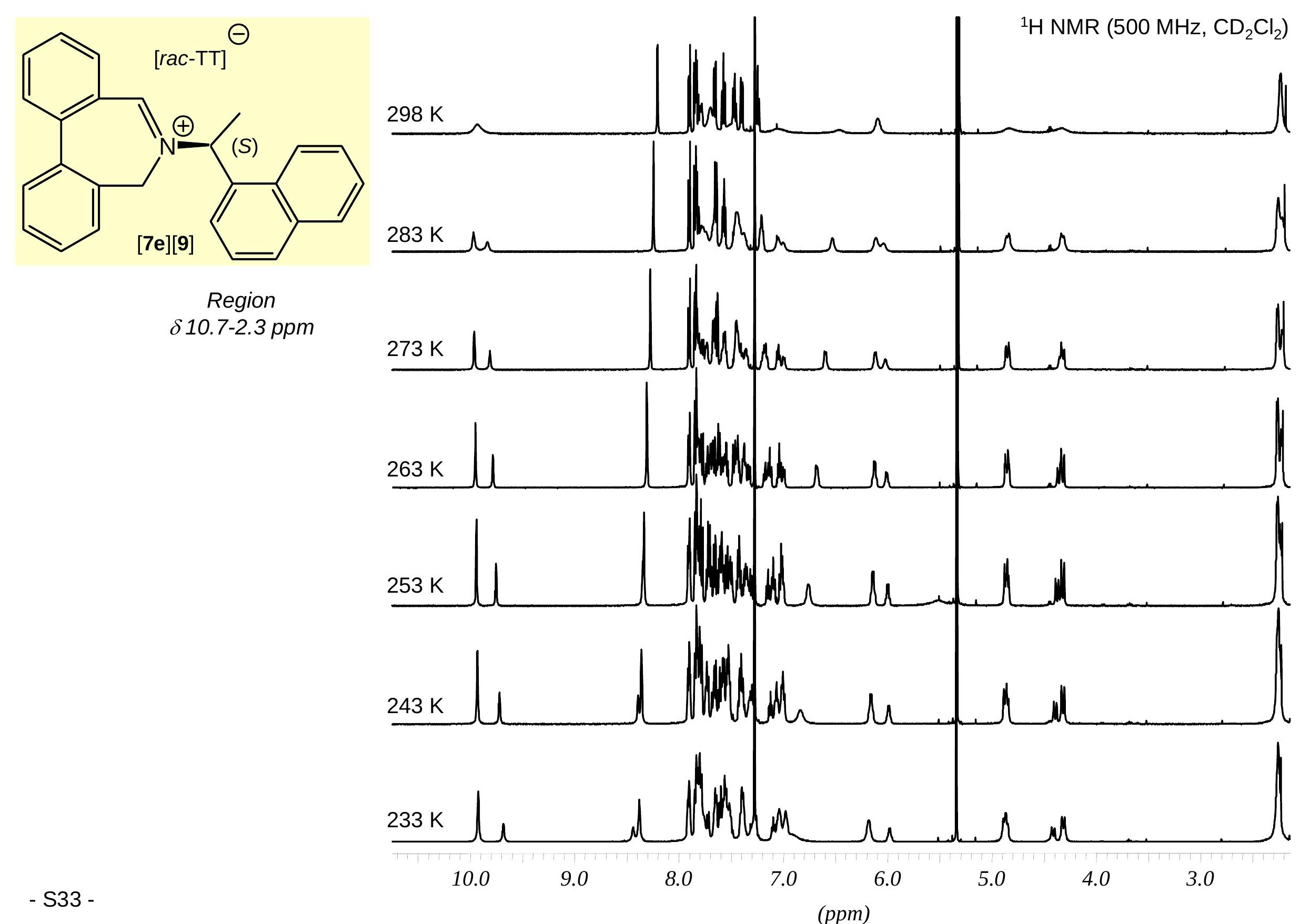



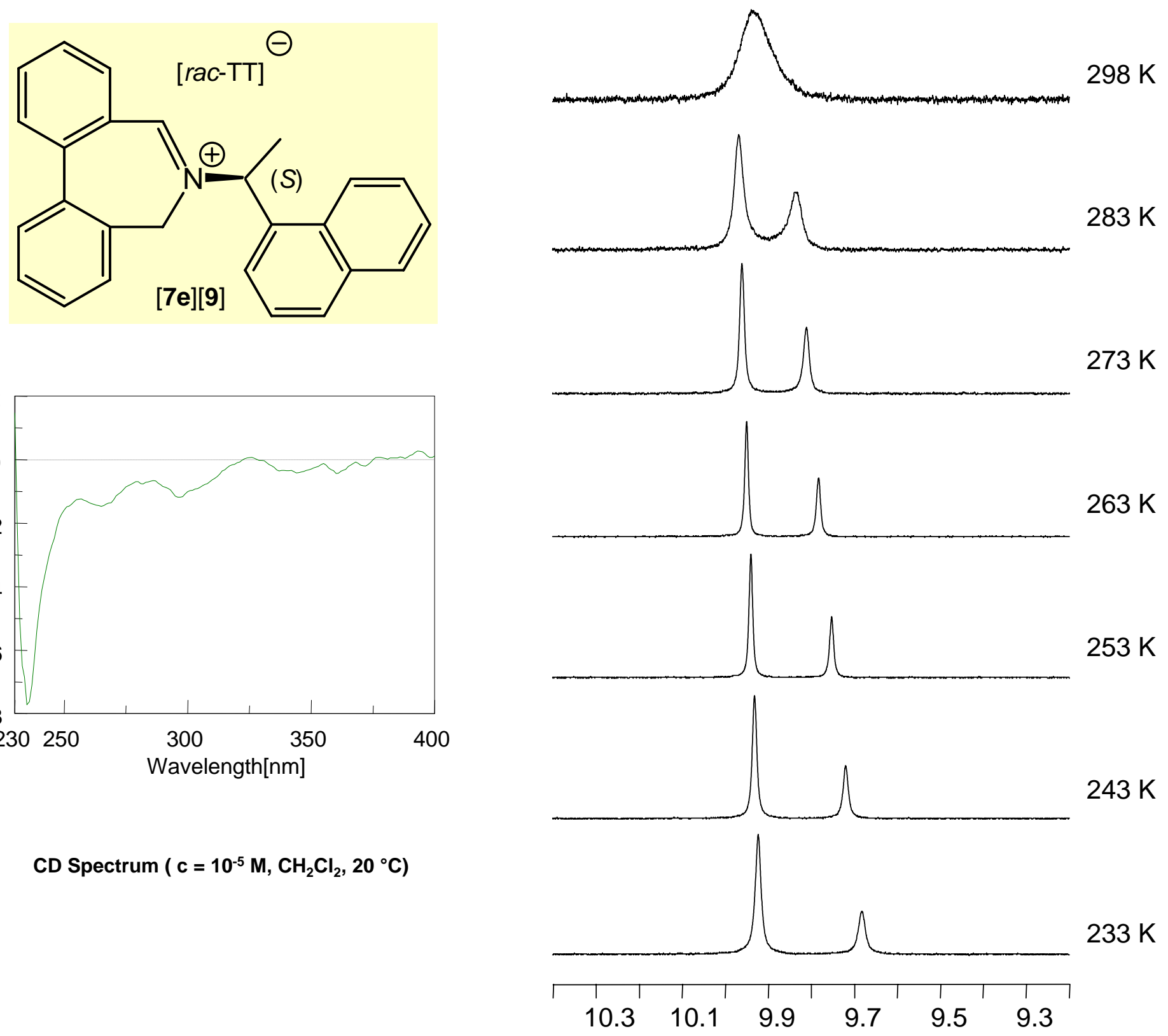

CD Spectrum ( $\mathrm{c}=10^{-5} \mathrm{M}, \mathrm{CH}_{2} \mathrm{Cl}_{2}, 20^{\circ} \mathrm{C}$ ) 
${ }^{1} \mathrm{H}$ NMR (400 MHz, DMSO)

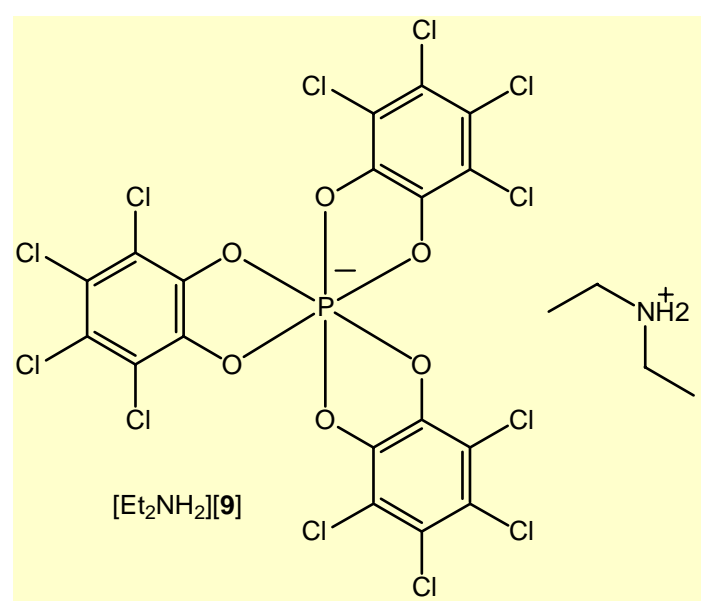

0
0
0
01
1
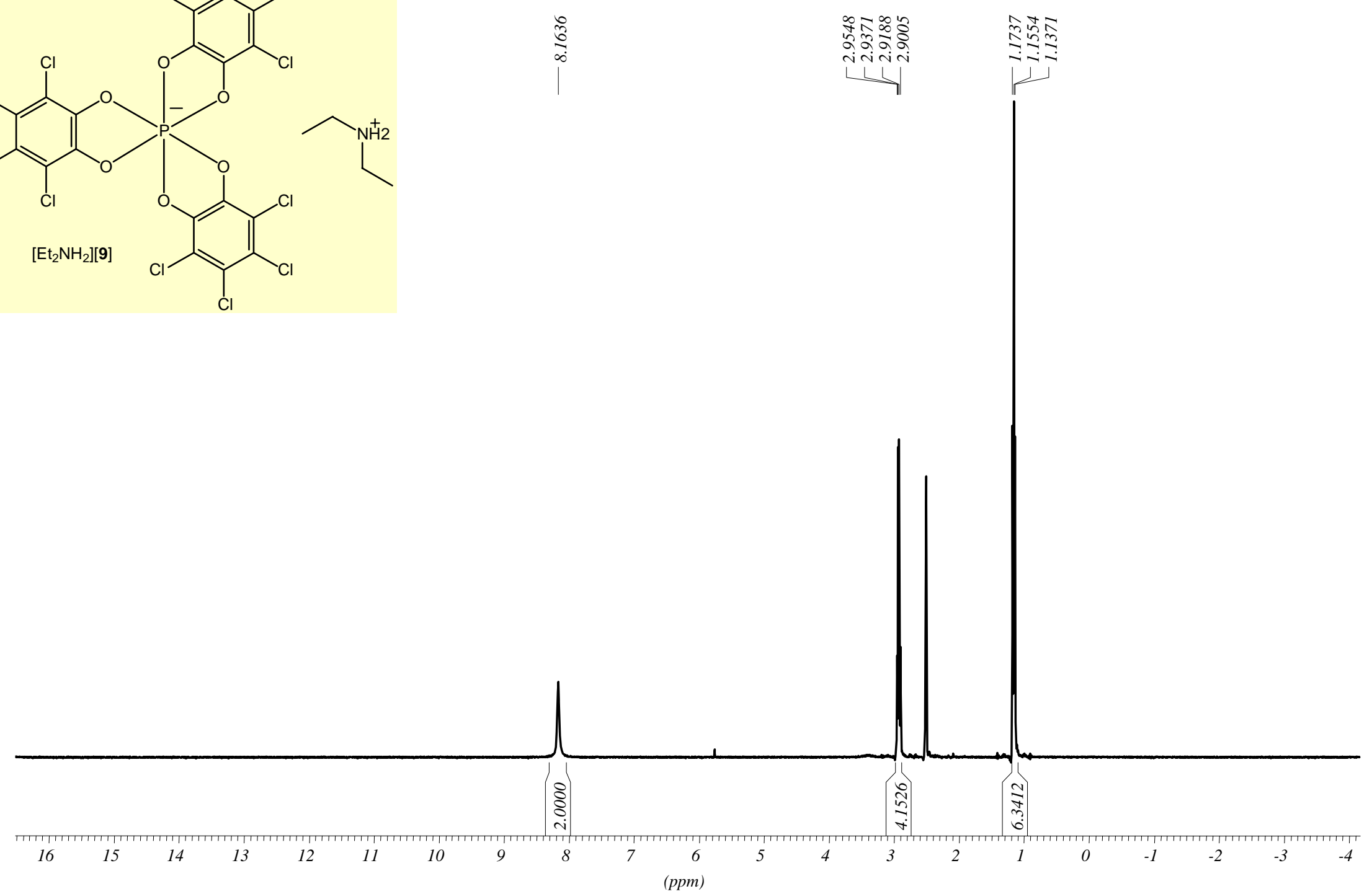

- S35 - 


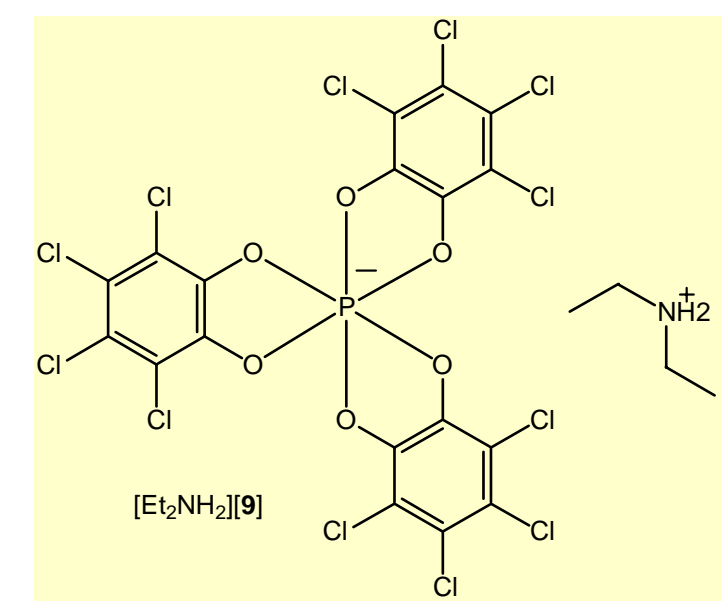

${ }^{13} \mathrm{C}$ NMR (100 MHz, DMSO)

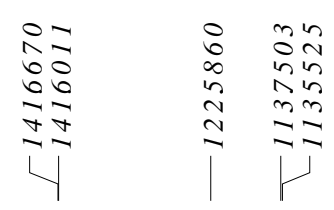

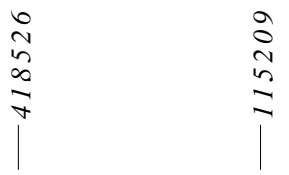

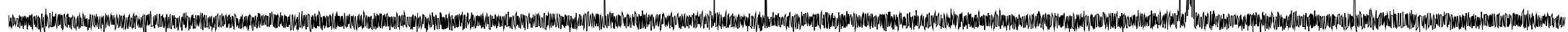

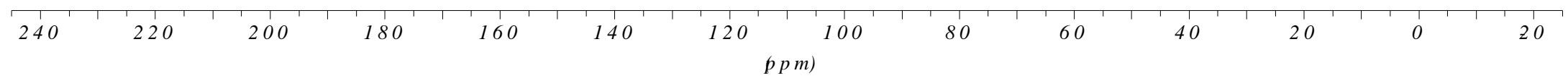




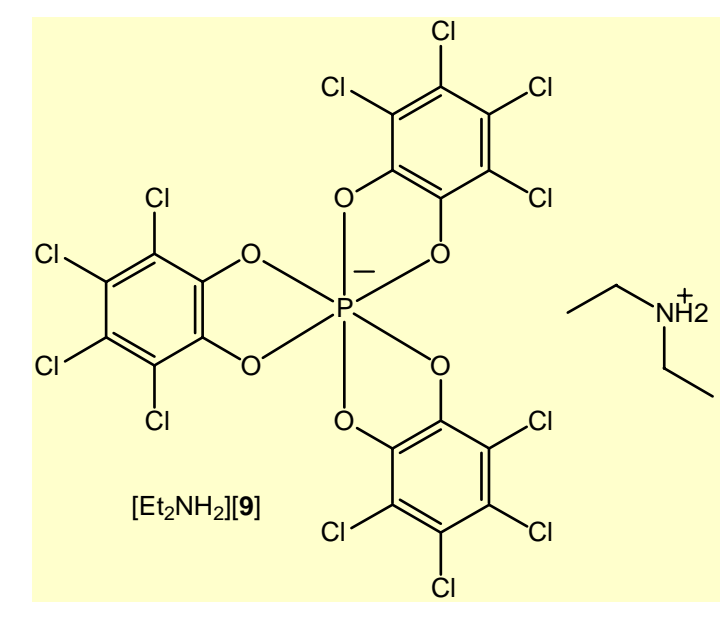

${ }^{31} \mathrm{P}$ NMR (162 MHz, $\mathrm{CDCl}_{3}$ )

$\infty$
$\infty$
$\infty$
$\infty$
$\infty$

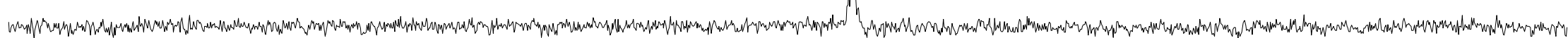

\begin{tabular}{|c|c|c|c|c|c|c|c|c|c|c|c|c|c|c|c|c|c|}
\hline-71 & -72 & -73 & -74 & -75 & -76 & -77 & -78 & -79 & $\begin{array}{c}-80 \\
(\mathrm{ppm})\end{array}$ & -81 & -82 & -83 & -8 & -85 & -86 & -8 & -88 \\
\hline
\end{tabular}


<smiles>C1=C(c2ccccc2)CCCC1</smiles>

12

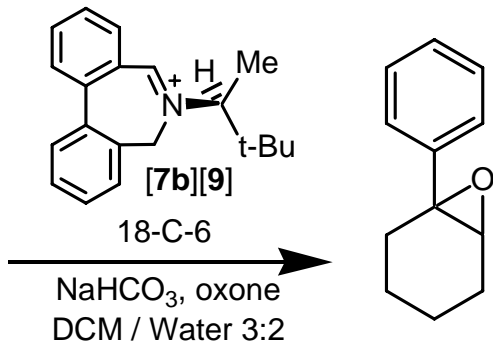

$2 \mathrm{~h}, 0^{\circ} \mathrm{C}$

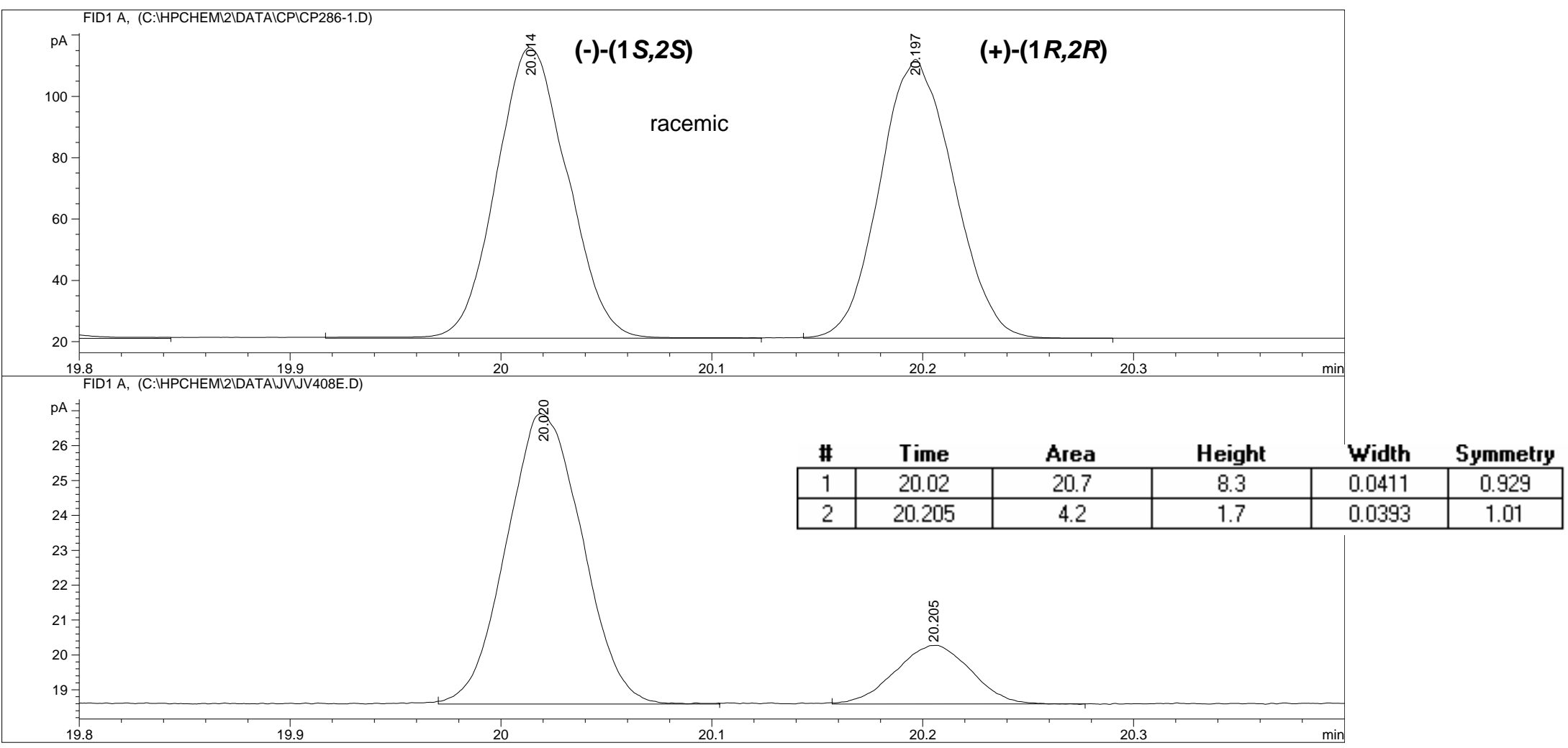

Determined by CSP-GC (Chiraldex Hydrodex $\beta-3 \mathrm{P}$ ), $\mathrm{T}_{\text {inj }} 250^{\circ} \mathrm{C}, \mathrm{P}=0.842 \mathrm{bar}$; 


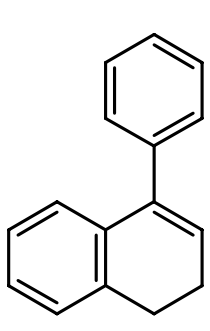

13

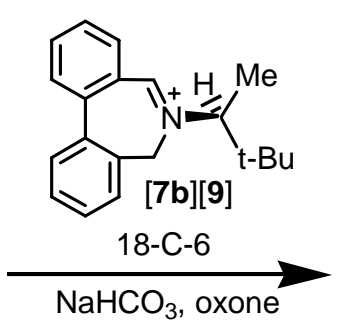

DCM / Water 3:2

$2 \mathrm{~h}, 0^{\circ} \mathrm{C}$

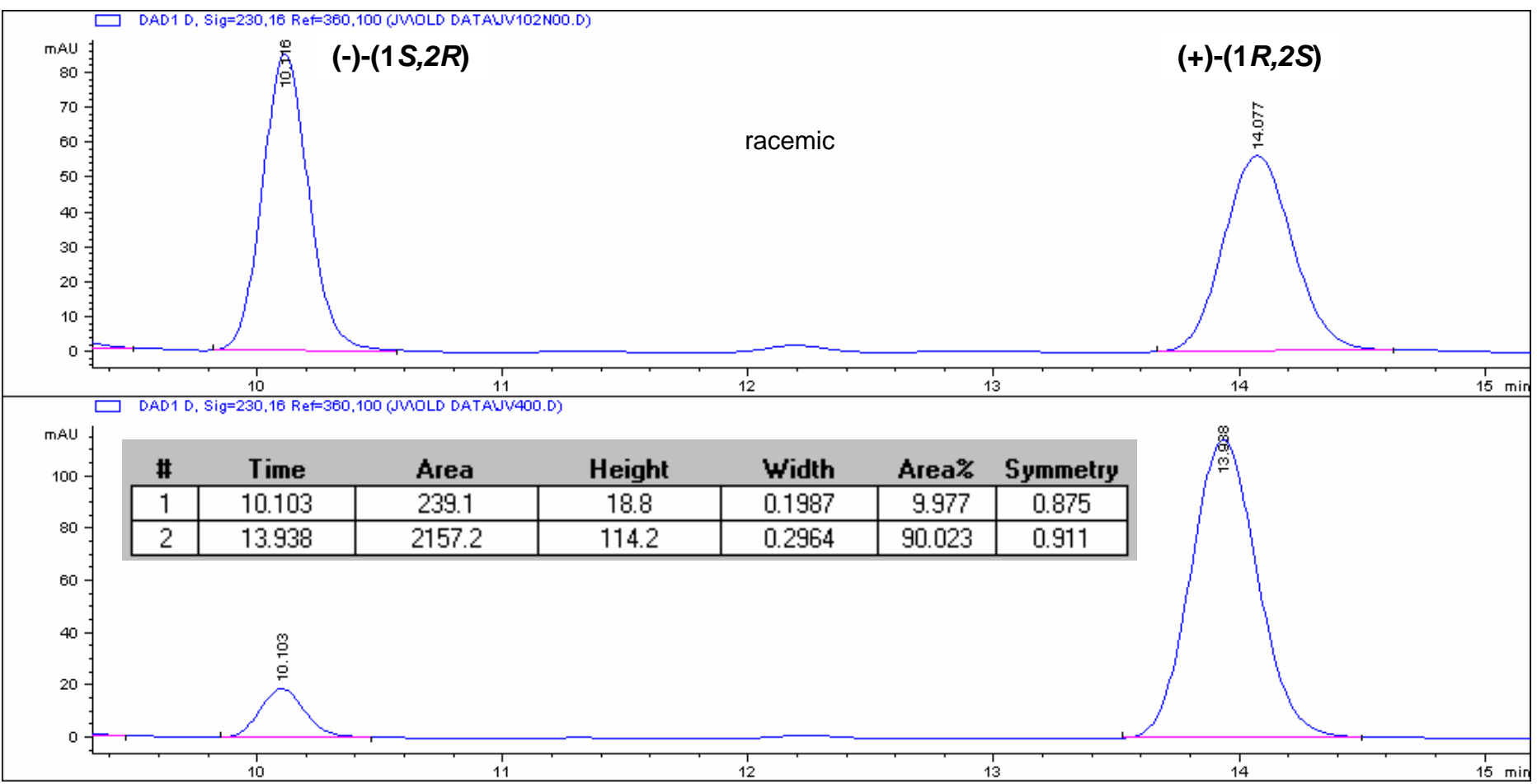

\section{A: Alkene, Ref: naphthalene}



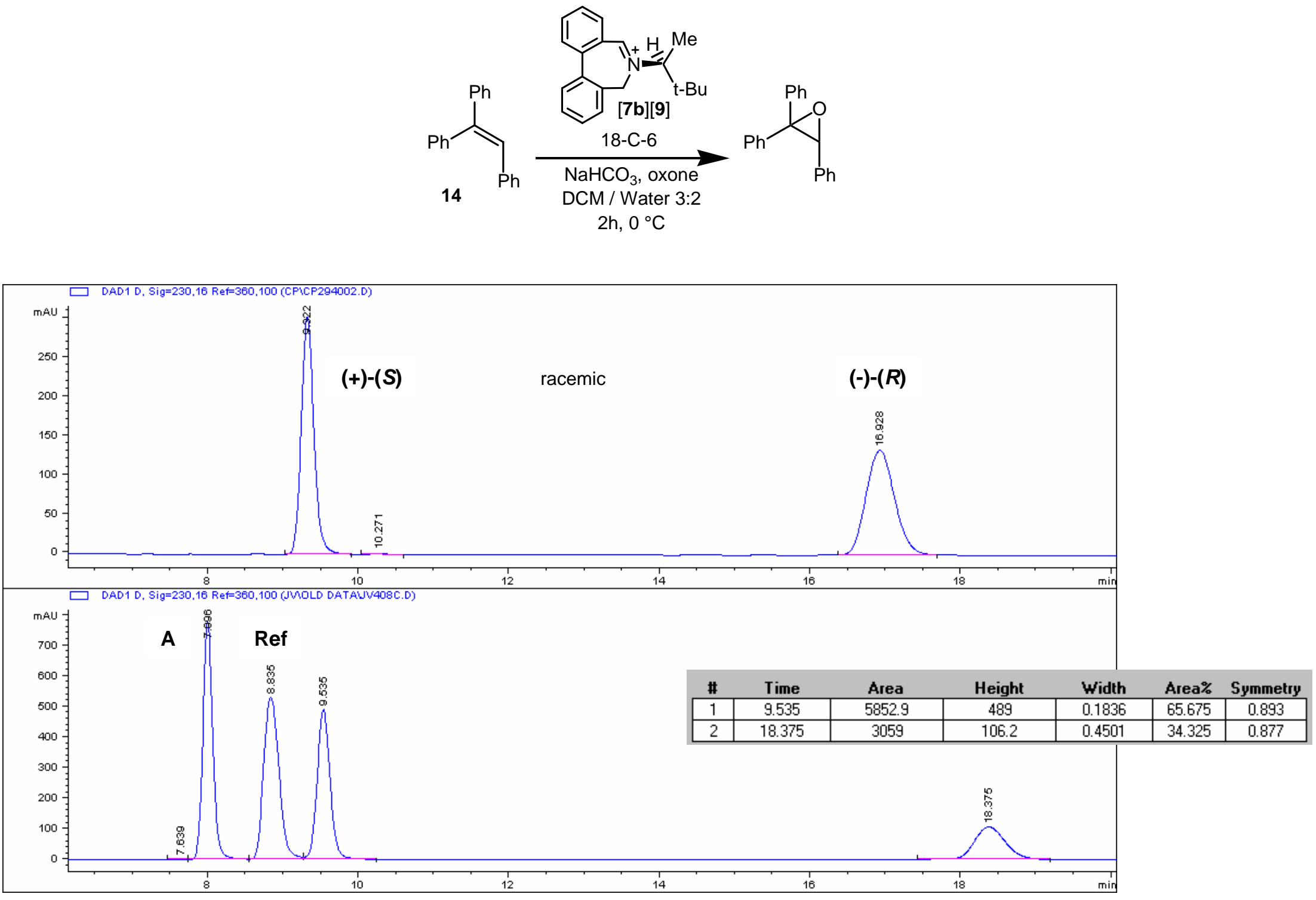

A: Alkene, Ref: naphthalene 

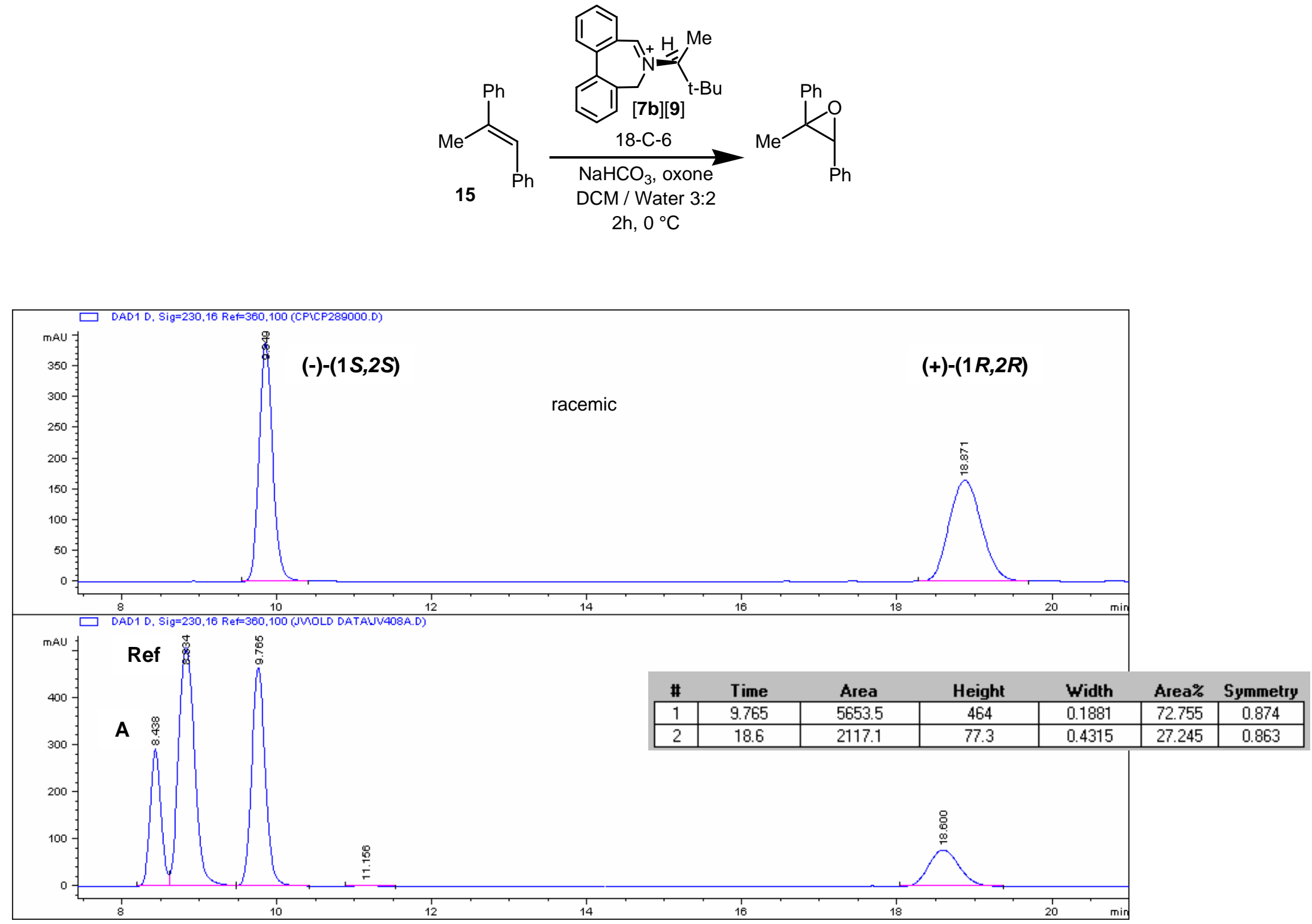

A: Alkene, Ref: naphthalene 

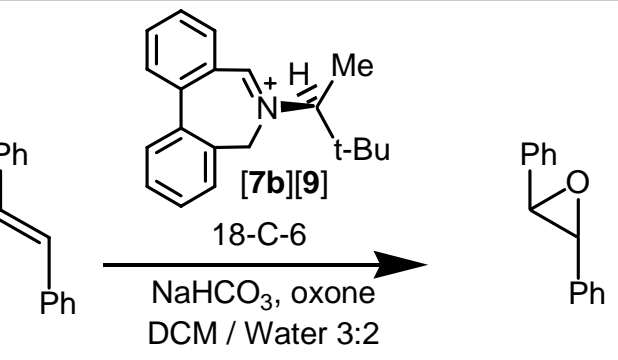

$2 \mathrm{~h}, 0^{\circ} \mathrm{C}$

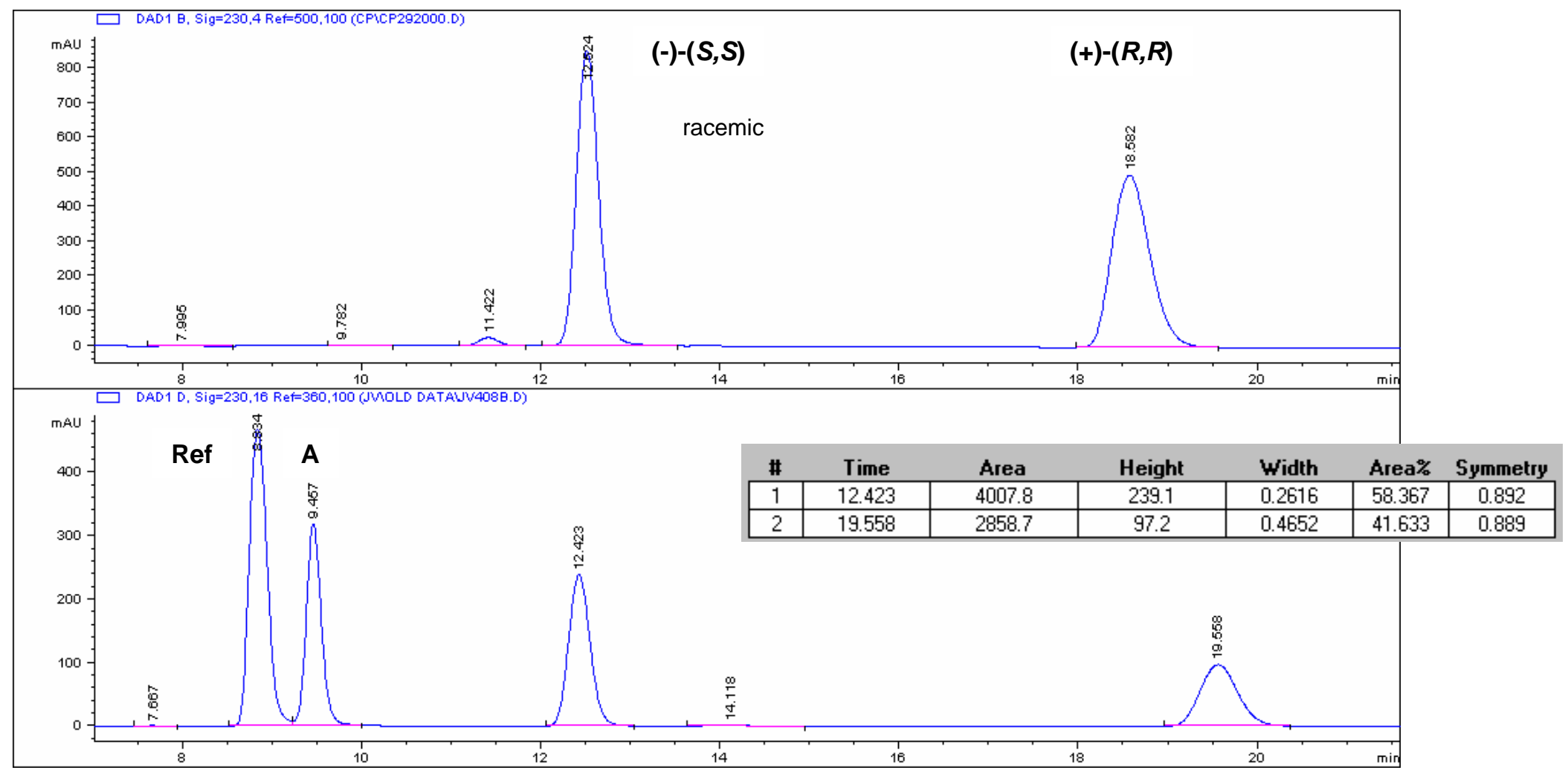

A: Alkene, Ref: naphthalene 